\title{
SIGN-CHANGING BLOW-UP FOR SCALAR CURVATURE TYPE EQUATIONS
}

\author{
FRÉDÉRIC ROBERT AND JÉRÔME VÉTOIS
}

\begin{abstract}
Given $(M, g)$ a compact Riemannian manifold of dimension $n \geq$ 3 , we are interested in the existence of blowing-up sign-changing families $\left(u_{\varepsilon}\right)_{\varepsilon>0} \in C^{2, \theta}(M), \theta \in(0,1)$, of solutions to

$$
\Delta_{g} u_{\varepsilon}+h u_{\varepsilon}=\left|u_{\varepsilon}\right|^{\frac{4}{n-2}-\varepsilon} u_{\varepsilon} \text { in } M
$$

where $\Delta_{g}:=-\operatorname{div}_{g}(\nabla)$ and $h \in C^{0, \theta}(M)$ is a potential. We prove that such families exist in two main cases: in small dimension $n \in\{3,4,5,6\}$ for any potential $h$ or in dimension $3 \leq n \leq 9$ when $h \equiv \frac{n-2}{4(n-1)}$ Scal $_{g}$. These examples yield a complete panorama of the compactness/noncompactness of critical elliptic equations of scalar curvature type on compact manifolds. The changing of the sign is necessary due to the compactness results of Druet [1] and Khuri-Marques-Schoen 19.
\end{abstract}

\section{INTRODUCTION}

Let $(M, g)$ be a smooth compact Riemannian manifold of dimension $n \geq 3$. Given $\theta \in(0,1)$, we consider solutions $u \in C^{2, \theta}(M)$ to the equation

$$
\Delta_{g} u+h u=|u|^{2^{\star}-2} u \quad \text { in } M
$$

where $h \in C^{0, \theta}(M), \Delta_{g}:=-\operatorname{div}_{g}(\nabla)$ is the Laplace-Beltrami operator, and $2^{\star}:=$ $\frac{2 n}{n-2}$. When $h \equiv \frac{n-2}{4(n-1)} \mathrm{Scal}_{g}\left(\mathrm{Scal}_{g}\right.$ being the scalar curvature of $\left.(M, g)\right)$, (10) is the Yamabe equation and rewrites

$$
\Delta_{g} u+c_{n} \operatorname{Scal}_{g} u=|u|^{2^{*}-2} u \text { in } M,
$$

where $c_{n}:=\frac{n-2}{4(n-1)}$. The conformal invariance of the Yamabe equation induces a dynamic that makes equations (11) and (2) unstable. Taking inspiration from the terminology introduced by R. Schoen [32, we say that equation (11) is compact (resp. positively compact) if for any family $\left(q_{\varepsilon}\right)_{\varepsilon} \in\left(2,2^{\star}\right]$ such that $q_{\varepsilon} \rightarrow 2^{\star}$ when $\varepsilon \rightarrow 0$ and for any family of functions (resp. positive functions) $\left(u_{\varepsilon}\right)_{\varepsilon} \in C^{2, \theta}(M)$ of solutions to

$$
\Delta_{g} u_{\varepsilon}+h u_{\varepsilon}=\left|u_{\varepsilon}\right|^{q_{\varepsilon}-2} u_{\varepsilon} \quad \text { in } M
$$

for $\varepsilon>0$ small, then a uniform bound on the Dirichlet energy $\left(\left\|\nabla u_{\varepsilon}\right\|_{2}\right)_{\varepsilon}$ implies the relative compactness of $\left(u_{\varepsilon}\right)_{\varepsilon}$ in $C^{2}(M)$, and therefore the convergence of a subfamily of $\left(u_{\varepsilon}\right)_{\varepsilon}$ in $C^{2}(M)$. Otherwise, we say that equation (11) is noncompact (resp. non positively-compact). A basic example of non compact equation is (2) on the canonical sphere $\left(\mathbb{S}^{n}, \mathrm{can}\right)$ : we refer to the second part of this section for (positive) compactness results for equations like (1).

Date: October 4th, 2012.

The authors are partially supported by the ANR grant ANR-08-BLAN-0335-01. 
We say that a family $\left(u_{\varepsilon}\right)_{\varepsilon>0} \in C^{2, \theta}(M)$ blows-up when $\varepsilon \rightarrow 0$ if $\lim _{\varepsilon \rightarrow 0}\left\|u_{\varepsilon}\right\|_{\infty}=$ $+\infty$. It is now well-known (see Struwe [34] for a description in Sobolev spaces and Druet-Hebey-Robert [14] for a description in $C^{0}$ ) that noncompactness is described by bubbles. In the present paper, we investigate the existence of families $\left(u_{\varepsilon}\right)_{\varepsilon} \in C^{2, \theta}(M)$ of sign-changing blowing-up solutions to the equation

$$
\Delta_{g} u_{\varepsilon}+h u_{\varepsilon}=\left|u_{\varepsilon}\right|^{2^{\star}-2-\varepsilon} u_{\varepsilon} \quad \text { in } M, \varepsilon>0 .
$$

In the sequel, we say that a blowing-up family $\left(u_{\varepsilon}\right)_{\varepsilon} \in C^{2, \theta}(M)$ is of type $\left(u_{0}-B\right)$ if there exists $u_{0} \in C^{2, \theta}(M)$ and a bubble $\left(B_{\varepsilon}\right)_{\varepsilon}$ (see definition (6) below) such that

$$
u_{\varepsilon}=u_{0}-B_{\varepsilon}+\mathrm{o}(1)
$$

where $\lim _{\varepsilon \rightarrow 0} \mathrm{O}(1)=0$ in $H_{1}^{2}(M)$, the completion of $C^{\infty}(M)$ for the norm $u \mapsto$ $\|u\|_{2}+\|\nabla u\|_{2}$. Our first result is the following:

Theorem 1.1 (dimensions $3 \leq n \leq 6$ and arbitrary potential). Let $(M, g)$ be $a$ smooth compact Riemannian manifold of dimension $3 \leq n \leq 6$ and let $h \in C^{0, \theta}(M)$ $(\theta \in(0,1))$ be such that $\Delta_{g}+h$ is coercive. Assume that there exists a nondegenerate solution $u_{0} \in C^{2, \theta}(M)$ to equation (10). In case $n=6$, assume in addition that $c_{n} \mathrm{Scal}_{g}-h<2 u_{0}$ in $M$. Then for $\varepsilon>0$ small, equation (41) admits a sign-changing solution $u_{\varepsilon}$ of type $\left(u_{0}-B\right)$. In particular, the family $\left(u_{\varepsilon}\right)_{\varepsilon>0}$ blows up as $\varepsilon \rightarrow 0$ and (1) is noncompact.

In full generality, it is not possible to construct positive blowing-up solutions to equation (41). Indeed, in addition to the assumptions of Theorem [1.1, if we assume that $h<c_{n} \mathrm{Scal}_{g}$, then (11) is positively compact (Druet [11 and the discussion below), and therefore any family of blowing-up solutions to (41) must be sign-changing. In the early reference [10, Ding proved the existence of infinitely many nonequivalent solutions to (2) on the canonical sphere, highlighting the diversity of the behavior of solutions to (1) depending on whether they are positive or negative.

The nondegeneracy assumption in Theorem 1.1 is necessary. We refer to Proposition 3.1 in Section 3 for the proof of necessity. However, the nondegeneracy assumption of Theorem 1.1 is generic in the sense that any degenerate solution to (1) can be approached by a solution of a slight perturbation of (1). We refer to Proposition 3.2 of Section 3 for the precise genericity statement.

The above theorem outlines a role of the geometry in dimension $n=6$. In higher dimension $n \geq 7$, the geometry of $(M, g)$ is more present. When the potential is strictly below the scalar curvature (that is $h<c_{n} \mathrm{Scal}_{g}$ ), equation (1) is compact for $n \geq 7$, at least in the locally conformally flat case (Vétois [36]). Conversely, if $h\left(x_{0}\right)>c_{n} \operatorname{Scal}_{g}\left(x_{0}\right)$ for some $x_{0} \in M$, then under some additional nondegeneracy assumption, equation (11) is non-compact when $n \geq 7$ (see Pistoia-Vétois [29] for general results). Our second result is in the case $h \equiv c_{n}$ Scal $_{g}$ :

Theorem 1.2 (dimensions $3 \leq n \leq 9$ and $\left.h \equiv c_{n} \operatorname{Scal}_{g}\right)$. Let $(M, g)$ be $a$ smooth compact Riemannian manifold of dimension $3 \leq n \leq 9$ with positive Yamabe invariant. Assume that there exists a nondegenerate positive solution $u_{0}>0$ to the Yamabe equation (2). Assume that $h \equiv c_{n} \mathrm{Scal}_{g}$. Then for $\varepsilon>0$ small, equation (4) admits a sign-changing solution $u_{\varepsilon}$ of type $\left(u_{0}-B\right)$. In particular, the family $\left(u_{\varepsilon}\right)_{\varepsilon>0}$ blows up as $\varepsilon \rightarrow 0$ and (2) is noncompact. 
Constructing positive blowing-up solutions is not possible in this context. Indeed, for $3 \leq n \leq 9$ and except for the canonical sphere, the scalar curvature equation (2) is positively compact (see Li-Zhu [25], Druet [12, Marques [26], Li-Zhang [22, 23, Khuri-Marques-Schoen [19, and the discussion below). We refer also to DruetHebey [13] and Druet-Hebey-Vétois [15] for the extension of compactness issues to stability issues.

The restriction of the dimensions in Theorem 1.2 is due to the geometry of the manifold. We refer to Subsection 2.1 in Section 2 for the extension of Theorem 1.2 to dimension $n=10$ in general and in any dimension in the locally conformally flat case.

Here again, it is natural to ask about the nondegeneracy assumption of a solution to the limit equation in Theorem 1.2 actually, it is both a necessary and a generic assumption. Concerning necessity, on the standard sphere (where all positive solutions to (2) are degenerate), it is not possible to construct blowing-up solutions of type $\left(u_{0}-B\right)$, see Proposition 3.1] in Section 3. However, it is proved in KhuriMarques-Schoen [19] that the nondegeneracy assumption is generic for the Yamabe equation (2), at least in dimensions $n \leq 24$, see Proposition 3.3 in Section 3 ,

Here is a brief overview of the positive compactness results known so far for equations like (1).

In 1987, Schoen [32 adressed the question of positive compactness of equation (2) for manifolds non conformally diffeomorphic to the canonical sphere $\left(\mathbb{S}^{n}\right.$, can) (say aspherical manifolds). The known results are the following: positive compactness holds for aspherical locally conformally flat manifolds (Schoen 32, 33]) and for arbitrary aspherical manifolds of dimension $3 \leq n \leq 24$ (Li-Zhu 25] $(n=3)$, Druet 12 ( $n \leq 5)$, Marques [26 $(n \leq 7)$, Li-Zhang 22 24 $(n \leq 11)$, KuhriMarques-Schoen [19] $(n \leq 24))$. But positive compactness does not hold in general in dimension $n \geq 25$ (There are blowing-up examples by Brendle [5] and BrendleMarques [6]). Combining these results with Theorem 1.1] we get that equation (2) is positively compact, but not compact, at least when $3 \leq n \leq 9$.

When $h \not \equiv c_{n} \mathrm{Scal}_{g}$, the situation is different. When $h<c_{n} \mathrm{Scal}_{g}$, Druet 11] proved that (3) is positively compact in dimension $n \geq 3$ (see also Li-Zhu [25] and DruetHebey-Vétois [15] for $n=3$ ). Conversely, in dimension $n \geq 4$, Micheletti-PistoiaVétois 27] and Pistoia-Vétois [29] proved that if $h$ is above $c_{n} \mathrm{Scal}_{g}$ somewhere, then, under some some nondegeneracy assumption, equation (1) is not positively compact. On the canonical sphere, there are blowing-up positive solutions with arbitrarily high energy when $h \equiv C t e>c_{n} \mathrm{Scal}_{\text {can }}$ (Chen-Wei-Yan 7 for $n \geq 5$ ). We refer to Esposito-Pistoia-Vétois [16] for blowing-up positive solutions in case of a potential $h$ depending on $\varepsilon$ and approaching $c_{n} \mathrm{Scal}_{g}$, and to Hebey-Wei [18] for the construction of multi-peak solutions on the three-sphere with a potential approaching constants arbitrarily larger than the scalar curvature. Here again, combining Druet [11] and Theorem 1.2 yields the following: when $h<c_{n} \mathrm{Scal}_{g}$ and $3 \leq n \leq 5$, equation (11) is positively compact, but not compact.

The proofs of Theorems 1.1 and 1.2 rely on a Lyapunov-Schmidt reduction. Over the past two decades, there has been intensive developments in Lyapunov-Schmidt reductions applied to critical elliptic equations. In addition to the references in the geometric context of a Riemannian manifold cited above, an early reference 
for single-bubble solutions is Rey [30]. Possible references on the construction of blowing-up solutions to equations like (4) by glueing a fixed function to bubbles are del Pino-Musso-Pacard-Pistoia [8, 9] and Guo-Li-Wei [17] (for the Yamabe equation on the canonical sphere) and Wei-Yan [37] (for a Lazer-McKenna type problem). The list of constributions above does not pretend to exhaustivity: we refer to the references of the above papers and also to the monograph [1] by AmbrosettiMalchiodi for further bibliographic complements. Our paper is organized as follows. In Section 2, we discuss extensions and generalizations of the above theorems. In Section 3, we discuss the nondegeneracy assumption. The finite dimensional reduction is performed in Section 4. The reduced problem is studied in Section 5 Theorems 1.1 and 1.2 are proved in Section 6. The proof of the error estimate is postponed to Section 7 .

Acknowledgements: The authors express their gratitude to Emmanuel Hebey and Lionel Bérard-Bergery for fruitful comments on this work.

\section{Miscellaneous extensions}

2.1. About the critical dimension $n=10$ in Theorem 1.2. As mentioned in the introduction, the method developed here fails to produce blowing-up solutions to (41) in higher dimension. Indeed, in dimensions $n \geq 7$, a term involving the Weyl tensor appear in the Taylor expansion (75) of the Lyapunov-Schmidt functional. In dimension $n<10$, this term is dominated by the contribution of $u_{0}$. In dimension $n=10$, there is a competition between the Weyl tensor and $u_{0}$, and one gets the following result:

Theorem 2.1 (dimension $n=10$ and $h \equiv c_{n} \operatorname{Scal}_{g}$ ). Let $(M, g)$ be a smooth compact Riemannian manifold of dimension $n=10$ with positive Yamabe invariant. Assume that there exists a nondegenerate positive solution to the Yamabe equation (21). Assume that $h \equiv c_{n} \mathrm{Scal}_{g}$ and that $u_{0}>\frac{5}{567}\left|\mathrm{Weyl}_{g}\right|_{g}^{2}$. Then for $\varepsilon>0$ small, equation (4) admits a sign-changing solution $u_{\varepsilon}$ of type $\left(u_{0}-B\right)$. In particular, the family $\left(u_{\varepsilon}\right)_{\varepsilon>0}$ blows up as $\varepsilon \rightarrow 0$.

In dimension $n>10$, the Weyl tensor dominates the contribution of $u_{0}$, and it is not possible to produce blowing-up solutions in general (see the explicit Taylor expansion (75) in Section (6). However, in the locally conformally flat case, that is when the Weyl tensor vanishes (at least in dimension larger than four), one gets the following result:

Theorem 2.2 (the locally conformally flat case in any dimension). Let $(M, g)$ be a smooth compact Riemannian manifold of dimension $n \geq 3$ with positive Yamabe invariant. Assume that there exists a nondegenerate positive solution to the Yamabe equation (2). Assume that $(M, g)$ is locally conformally flat and that $h \equiv c_{n}$ Scal $_{g}$. Then for $\varepsilon>0$ small, equation (4) admits a sign-changing solution $u_{\varepsilon}$ of type $\left(u_{0}-B\right)$. In particular, the family $\left(u_{\varepsilon}\right)_{\varepsilon>0}$ blows up as $\varepsilon \rightarrow 0$.

Examples of manifolds and metrics satisfying the hypothesis of Theorem 2.2 are in Proposition 3.4. As stated in Theorems 2.1 and 2.2, the solutions we construct change sign. Here again, it is natural to ask if there exist positive blowing-up solutions to (41) under the assumptions of Theorems 2.1 and 2.2. The answer is negative. Indeed, it follows from positive compactness theorems (Khuri-MarquesSchoen [19] and Schoen [32,33]) that positive blowing-up solutions to equation (4) 
do not exist in the locally conformally flat and aspherical case. A consequence of the above results is that the Yamabe equation (2) is positively compact, but not compact in the context of Theorems 2.1 and 2.2 .

2.2. Positive blowing-up solutions in dimension $n=6$. In this subsection, we focus on positive solutions to (4). A direct offshot of the techniques developed for the proof of Theorem 1.1 yields the following result:

Theorem 2.3 (positive solutions in dimension $n=6)$. Let $(M, g)$ be a smooth compact Riemannian manifold of dimension $n=6$ and let $h \in C^{0, \theta}(M)(\theta \in(0,1))$ be such that $\Delta_{g}+h$ is coercive. Assume that there exists a nondegenerate solution $u_{0} \in C^{2, \theta}(M)$ to equation (10) and that

$$
h-c_{6} \operatorname{Scal}_{g}>2 u_{0}>0 \text { in } M .
$$

Then for $\varepsilon>0$ small, equation (44) admits a positive solution $u_{\varepsilon}>0$ such that $u_{\varepsilon}=u_{0}+B_{\varepsilon}+o(1)$, where $\left(B_{\varepsilon}\right)_{\varepsilon}$ is a bubble and $\lim _{\varepsilon \rightarrow 0} o(1)=0$ in $H_{1}^{2}(M)$.

This result is a complement to a specific 6-dimensional result: Druet ([11] and private communication) showed that blow-up for positive solutions to (4) with bounded energy necessarily occurs at points $x \in M$ such that $\left(h-c_{6} \operatorname{Scal}_{g}\right)(x) \geq$ $2 u_{0}(x)$. Dimension six is critical when considering positive blowing-up solutions with nontrivial weak limit $u_{0}>0$. More precisely, the blow-up analysis shows that there is balance between the contributions of $u_{0}$ and $h-c_{n}$ Scal $_{g}$ : one of the terms dominates the other when $n \neq 6$, and they compete at the same growth when $n=6$. We refer to the Taylor expansion (45) and to 11] to outline this phenomenon. We refer to Druet-Hebey [13] for an extensive discussion on dimension six.

2.3. Prescription of the blow-up point. The above theorems show the existence of blowing-up families of solutions, but the blow-up point (that is the point where the bubble is centered) is not prescribed. The only information obtained from the construction is that blow-up occurs at a minimum point of $u_{0}$ (for Theorems 1.1 and 1.2 when $n \neq 6$ ) or of $u_{0}-\left(c_{n} \mathrm{Scal}_{g}-h\right) / 2$ (for Theorem 1.1 when $n=6$ ). Prescribing the location of the blow-up point of the bubbles requires additional informations. We define $\Phi: M \rightarrow \mathbb{R}$ as follows:

$$
\Phi:= \begin{cases}u_{0}+\frac{1}{2}\left(h-c_{n} \operatorname{Scal}_{g}\right) \mathbf{1}_{n=6} & \text { in Theorems 1.1, 1.2 and } 2.2 \\ u_{0}-\frac{5}{567} \mid \text { Weyl }\left._{g}\right|_{g} ^{2} & \text { in Theorem 2.1 } \\ \frac{1}{2}\left(h-c_{6} \text { Scal }_{g}\right)-u_{0} & \text { in Theorem 2.3 }\end{cases}
$$

Our prescription result is the following:

Theorem 2.4 (Prescription of the blow-up point). In addition to the hypothesis in Theorems 1.1, 1.2, 2.1, 2.2, and 2.3, assume that there exists $\xi_{0} \in M$ which is a strict local minimum point of $\Phi$ with $\Phi\left(\xi_{0}\right)>0$. Then the conclusions of the above theorems hold with the extra information that the bubbles are centered at a family $\left(\xi_{\varepsilon}\right)_{\varepsilon} \in M$ such that $\lim _{\varepsilon \rightarrow 0} \xi_{\varepsilon}=\xi_{0}$.

In case $h \in C^{1}(M)$ and there exists $\xi_{0} \in M$ which is a $C^{1}$-stable critical point of $\Phi$ with $\Phi\left(\xi_{0}\right)>0$, the same conclusion holds with the convergence (45) holding in $C^{1}$. 


\section{Discussion On THE DEGENERATE CASE}

In the sequel, we say that $\left(B_{\varepsilon}\right)_{\varepsilon}$ is a bubble if there exists a family $\left(x_{\varepsilon}\right)_{\varepsilon} \in M$ and a family $\left(\mu_{\varepsilon}\right)_{\varepsilon} \in \mathbb{R}_{>0}$ such that $\lim _{\varepsilon \rightarrow 0} \mu_{\varepsilon}=0$ and

$$
B_{\varepsilon}(x):=\left(\frac{\sqrt{n(n-2)} \mu_{\varepsilon}}{\mu_{\varepsilon}^{2}+d_{g}\left(x, x_{\varepsilon}\right)^{2}}\right)^{\frac{n-2}{2}} \text { for all } x \in M .
$$

In this situation, we say that the bubble is centered at $\left(x_{\varepsilon}\right)_{\varepsilon}$. We say that a solution $u_{0} \in C^{2, \theta}(M)$ to

$$
\Delta_{g} u_{0}+h u_{0}=u_{0}^{2^{\star}-1} \quad \text { in } M
$$

is nondegenerate if the linearization of the equation has a trivial kernel, that is

$$
K_{h, u_{0}}:=\left\{\varphi \in C^{2, \theta}(M) / \Delta_{g} \varphi+h \varphi=\left(2^{\star}-1\right)\left|u_{0}\right|^{2^{\star}-2} \varphi\right\}=\{0\} .
$$

Theorems 1.1 and 1.2 require the assumption that $u_{0}$ is a nondegenerate solution to (7). In this section, we prove that this is a necessary assumption, and that it is generic.

3.1. The conformal geometric equation and necessity of the nondegeneracy assumption. Let $(M, g)$ be a compact Riemannian manifold of dimension $n \geq 3$ with positive Yamabe invariant. Up to a conformal change of metric, it follows from the resolution of the Yamabe problem that we can assume that the scalar curvature $\mathrm{Scal}_{g}$ is a positive constant, and we consider $u_{0, g}:=\left(c_{n} \mathrm{Scal}_{g}\right)^{1 /\left(2^{\star}-2\right)}$ the only positive constant solution to the Yamabe equation

$$
\Delta_{g} u_{0, g}+c_{n} \operatorname{Scal}_{g} u_{0, g}=u_{0, g}^{2^{\star}-1} \text { in } M .
$$

As is easily checked, in this situation,

$$
K_{c_{n} \mathrm{Scal}_{g}, u_{0, g}}=\left\{\varphi \in C^{2}(M) / \Delta_{g} \varphi=\frac{\operatorname{Scal}_{g}}{n-1} \varphi\right\},
$$

where the kernel is defined in (18). Therefore

$$
u_{0, g} \text { is a nondegenerate solution to (9) } \Leftrightarrow \frac{\operatorname{Scal}_{g}}{n-1} \notin \operatorname{Spec}\left(\Delta_{g}\right),
$$

where Spec $\left(\Delta_{g}\right)$ is the nonnegative spectrum of $\Delta_{g}$. We define the Yamabe invariant by

$$
\mu_{[g]}(M):=\inf _{g^{\prime} \in[g]} \frac{\int_{M} \operatorname{Scal}_{g^{\prime}} d v_{g^{\prime}}}{\operatorname{Vol}_{g^{\prime}}(M)^{\frac{n-2}{n}}},
$$

where $[g]$ is the conformal class of $g$ and $d v_{g}$ is the Riemannian element of volume. The Yamabe invariant $\mu_{[g]}(M)$ is positive iff the operator $\Delta_{g^{\prime}}+c_{n} \mathrm{Scal}_{g^{\prime}}$ is coercive for all $g^{\prime} \in[g]$. It is well known that if $g$ is a Yamabe metric (that is a minimizer of the Yamabe functional (10)), one has that $\frac{\mathrm{Scal}_{g}}{n-1} \leq \lambda_{1}\left(\Delta_{g}\right)$, the first nonzero eigenvalue of $\Delta_{g}$. Note that equality is achieved on the canonical sphere $\left(\mathbb{S}^{n}, \mathrm{can}\right)$. More generally, any positive solution to the Yamabe equation on the canonical sphere is a Yamabe metric and is degenerate.

The following result shows that the conclusion of Theorems 1.1 and 1.2 do not hold on the standard sphere (where positive solutions to the Yamabe equations are all degenerate): 
Proposition 3.1. There does not exist any family of functions $\left(u_{\varepsilon}\right)_{\varepsilon} \in C^{2, \theta}\left(\mathbb{S}^{n}\right)$ of type $\left(u_{0}-B\right)$ to the equation

$$
\Delta_{\text {can }} u_{\varepsilon}+c_{n} \text { Scal }_{\text {can }} u_{\varepsilon}=\left|u_{\varepsilon}\right|^{2^{\star}-2-\varepsilon} u_{\varepsilon} \quad \text { in } M
$$

for all $\varepsilon \in\left(0,2^{\star}\right)$.

Proof. We argue by contradiction and assume the existence of a family $\left(u_{\varepsilon}\right)_{\varepsilon} \in$ $C^{2, \theta}\left(\mathbb{S}^{n}\right)$ of the form (5) of solutions to equation (11). Multiplying (11) by the bubble $B_{\varepsilon}$ and integrating by parts yield $\mu_{\varepsilon}^{\varepsilon} \rightarrow 1$ when $\varepsilon \rightarrow 0$. Fix $\phi \in \Lambda_{1}\left(\mathbb{S}^{n}\right.$, can), the set of eigenfunctions of $\lambda_{1}\left(\Delta_{\text {can }}\right)=n$, the first nonzero eigenvalue of $\Delta_{\text {can }}$ on $\mathbb{S}^{n}$ : indeed, see for instance Berger-Gauduchon-Mazet [3], we have that $\Lambda_{1}\left(\mathbb{S}^{n}\right.$, can $)=$ $\left\{l_{\mid \mathbb{S}^{n}} / l: \mathbb{R}^{n+1} \rightarrow \mathbb{R}\right.$ linear $\}$. It follows from Kazdan-Warner [20] that

$$
\int_{\mathbb{S}^{n}} \Delta_{\text {can }} u_{\varepsilon}\left\langle\nabla \phi, \nabla u_{\varepsilon}\right\rangle_{\text {can }} d v_{\text {can }}=\frac{n-2}{2 n} \int_{\mathbb{S}^{n}} \Delta_{\text {can }} \phi\left|\nabla u_{\varepsilon}\right|_{\text {can }}^{2} d v_{\text {can }} .
$$

Using equation (11) and integrating by parts yields

$$
\varepsilon \int_{\mathbb{S}^{n}} \phi\left|u_{\varepsilon}\right|^{2^{\star}-\varepsilon} d v_{\mathrm{can}}=0
$$

Letting $\varepsilon \rightarrow 0$ and using (6), (5), and (15) yields, up to a subsequence,

$$
\int_{\mathbb{S}^{n}} \phi u_{0}^{2^{\star}} d v_{\mathrm{can}}+\left(\int_{\mathbb{R}^{n}} U^{2^{\star}} d x\right) \phi\left(x_{0}\right)=0
$$

where $x_{0} \in \mathbb{S}^{n}$. Passing to the weak limit in (11) when $\varepsilon \rightarrow 0$ implies that $u_{0}$ is a positive solutions to the Yamabe equation on $\mathbb{S}^{n}$. It then follows from Obata 28] that $\int_{\mathbb{S}^{n}} u_{0}^{2^{\star}} d v_{\text {can }}=\int_{\mathbb{R}^{n}} U^{2^{\star}} d x$. Taking $\phi \in \Lambda_{1}\left(\mathbb{S}^{n}\right.$, can) such that $\min \phi=\phi\left(-x_{0}\right) \neq 0$ in the above equation yields a contradiction since $\phi \not \equiv 0$. This ends the proof of Proposition 3.1 .

3.2. Genericity of the nondegeneracy assumption. The following proposition shows that the nondegeneracy hypothesis of Theorem 1.1 is generic:

Proposition 3.2. Let $h \in C^{0, \theta}(M)$ and let $u_{0} \in C^{2, \theta}(M)$ be a positive solution to $\Delta_{g} u_{0}+h u_{0}=u_{0}^{2^{\star}-1}$. Fix $\nu>0$. Then there exist $\tilde{h}_{\nu} \in C^{0, \theta}(M)$ and $\tilde{u}_{0, \nu} \in C^{2, \theta}(M)$ such that $\left\|h-\tilde{h}_{\nu}\right\|_{C^{0, \theta}}+\left\|u_{0}-\tilde{u}_{0, \nu}\right\|_{C^{2, \theta}}<\nu$ and $\tilde{u}_{0, \nu}>0$ is a nondegenerate solution to $\Delta_{g} \tilde{u}_{0, \nu}+\tilde{h}_{\nu} \tilde{u}_{0, \nu}=\tilde{u}_{0, \nu}^{2^{\star}-1}$ in $M$.

Proof. We define

$$
\mu_{\eta}:=\inf _{u \in H_{1}^{2}(M) \backslash\{0\}} \frac{\int_{M}\left(|\nabla u|_{g}^{2}+\left(h-\left(2^{\star}-1\right) u_{0}^{2^{\star}-2}-\eta\right) u^{2}\right) d v_{g}}{\left(\int_{M}|u|^{2^{\star}} d v_{g}\right)^{\frac{2}{2^{\star}}}}
$$

for all $\eta \geq 0$. Testing the functional on $u_{0}$ yields $\mu_{\eta}<0$ for all $\eta \geq 0$. As is easily checked, $\lim _{\eta \rightarrow 0} \mu_{\eta}=\mu_{0}<0$. Standard variational arguments yield the existence of a positive minimizer $w_{\eta} \in C^{2, \theta}(M)$ for $\mu_{\eta}$ such that $\Delta_{g} w_{\eta}+(h-$ $\left.\left(2^{\star}-1\right) u_{0}^{2^{\star}-2}-\eta\right) w_{\eta}=-\left(2^{\star}-2\right) w_{\eta}^{2^{\star}-1}$ in $M$ for all $\eta>0$; moreover, the family $\left(w_{\eta}\right)_{\eta \geq 0}$ is relatively compact in $C^{2}(M)$. Since $u_{0}$ is the only positive solution to the equation $\Delta_{g} v+\left(h-\left(2^{\star}-1\right) u_{0}^{2^{\star}-2}\right) v=-\left(2^{\star}-2\right) v^{2^{\star}-1}$ (let $w$ be the quotient of two positive solutions and estimate $\Delta_{g} w$ at extremal points of $w$ ), one gets that $\lim _{\eta \rightarrow 0} w_{\eta}=u_{0}$ in $C^{2}(M)$, and then $C^{2, \theta}(M)$ by elliptic regularity. One defines $h_{\eta}:=h-\left(2^{\star}-1\right)\left(u_{0}^{2^{\star}-2}-w_{\eta}^{2^{\star}-2}\right)-\eta$. Then $\Delta_{g} w_{\eta}+h_{\eta} w_{\eta}=w_{\eta}^{2^{\star}-1}$ in $M$ and spectral theory yields the existence of $\eta_{0}>0$ such that $K_{h_{\eta}, w_{\eta}}=\{0\}$ for all $\eta \in\left(0, \eta_{0}\right)$. 
The conclusion of the proposition follows from taking $\tilde{u}_{0, \nu}:=w_{\eta}$ and $\tilde{h}_{\nu}:=h_{\eta}$ for $\eta>0$ small enough.

We now focus on the geometric case, that is Theorem 1.2 We adopt the terminology of Khuri-Marques-Schoen [19]: given $M$ a compact manifold, $g_{0}$ a background Riemannian metric on $M$, and $\omega$ a volume form on $M$, to each class $c \in \mathcal{C}:=$ $\{$ Conformal classes of Riemannian metrics on $M$ \}, we associate the unique metric $g \in c$ for which the Riemannian $n$-volume form is $\omega$. The $C^{k, \theta}$-distance between two classes is defined as the $C^{k, \theta}$-distance between their representatives via this analogy. The following result holds:

Proposition 3.3 (Khuri-Marques-Schoen [19]). There exists $\mathcal{O} \subset \mathcal{C}$ an open dense set such that for all $c \in \mathcal{O}$, there exists exactly a finite nonzero number of metrics $g \in c$ (up to homothetic transformations) such that the constant positive function $u_{0, g}$ is nondegenerate.

In other words, up to a perturbation in the conformal class, the hypothesis of Theorem 1.2 holds.

3.3. A family of nondegenerate geometric solutions in the locally conformally flat case. We exhibit here a situation in which the nondegeneracy assumption is satisfied for the geometric equation in the locally conformally flat case (see Theorem 2.2). For all $k \geq 1$ and $t>0$, $\left(\mathbb{S}^{k}(t)\right.$, can) is the canonical sphere ot radius $t$ in $\mathbb{R}^{k+1}$ :

Proposition 3.4. Let $M_{r}:=\mathbb{S}^{1}(r) \times \mathbb{S}^{n-1}$ be endowed with its canonical product metric $g_{r}$, where $r>0$. Then $\left(M_{r}, g_{r}\right)$ is locally conformally flat with positive constant scalar curvature. Moreover, for any $r \notin\left\{\frac{i}{\sqrt{n-2}} / i \in \mathbb{Z}_{>0}\right\}$, the positive constant solution to the Yamabe equation is nondegenerate.

Proof. Recall that on the canonical sphere $\mathbb{S}^{k}(t)$, the spectrum of the Laplacian is $\left\{\frac{i(k+i-1)}{t^{2}} / i \in \mathbb{Z}_{\geq 0}\right\}$ (see [3]). Then the spectrum of $\Delta_{g_{r}}$ is $\left\{\frac{i^{2}}{r^{2}}+j(n-2+\right.$ $\left.j) / i, j \in \mathbb{Z}_{\geq 0}\right\}$. Independently, the scalar curvature is $\operatorname{Scal}_{g_{r}}:=(n-1)(n-2)$. As a consequence,

$$
\frac{\operatorname{Scal}_{g_{r}}}{n-1} \notin \operatorname{Spec}\left(\Delta_{g_{r}}\right) \Leftrightarrow r \notin\left\{\frac{i}{\sqrt{n-2}} / i \in \mathbb{Z}_{>0}\right\} .
$$

In addition, it is standard that the product of a one-dimensional circle with a space form is locally conformally flat.

\section{Finite dimensional Reduction}

Since the operator $\Delta_{g}+h$ is coercive, the Sobolev space $H_{1}^{2}(M)$ is endowed with the scalar product $\langle\cdot, \cdot\rangle_{h}$ defined by

$$
\langle u, v\rangle_{h}=\int_{M}\langle\nabla u, \nabla v\rangle_{g} d v_{g}+\int_{M} h u v d v_{g}
$$

for all $u, v \in H_{1}^{2}(M)$. We let $\|\cdot\|_{h}$ be the norm induced by $\langle\cdot, \cdot\rangle_{h}$ : this norm is equivalent to the standard norm on $H_{1}^{2}(M)$. We let $i^{*}: L^{\frac{2 n}{n+2}}(M) \rightarrow H_{1}^{2}(M)$ be such that for any $w$ in $L^{\frac{2 n}{n+2}}(M)$, the function $u=i^{*}(w)$ in $H_{1}^{2}(M)$ is the unique solution of the equation $\Delta_{g} u+h u=w$ in $M$. We then rewrite equation (4) as

$$
u=i^{*}\left(f_{\varepsilon}(u)\right), \quad u \in H_{1}^{2}(M),
$$


where $f_{\varepsilon}(u):=|u|^{2^{*}-2-\varepsilon} u$.

In case $(M, g)$ is locally conformally flat, it follows from the compactness of $M$ that there exists $r_{0} \in\left(0, i_{g}(M)\right)$ (where $i_{g}(M)>0$ is the injectivity radius of $(M, g)$ ) such that for any point $\xi$ in $M$, there exists $\Lambda_{\xi} \in C^{\infty}(M)$ such that the conformal metric $g_{\xi}=\Lambda_{\xi}^{4 /(n-2)} g$ is flat in $B_{\xi}\left(r_{0}\right)$ and $i_{g_{\xi}}(M)>r_{0}$. As is easily seen, the functions $\Lambda_{\xi}$ can be chosen smooth with respect to $\xi$ and such that $\Lambda_{\xi}(\xi)=1$. If the manifold is not locally conformally flat, then we let $\Lambda_{\xi}(x)=1$ for all points $x$ and $\xi$ in $M$, and we fix $r_{0} \in\left(0, i_{g}(M)\right)$ arbitrarily. We let $\chi$ be a smooth cutoff function such that $0 \leq \chi \leq 1$ in $\mathbb{R}, \chi=1$ in $\left[-r_{0} / 2, r_{0} / 2\right]$, and $\chi=0$ in $\mathbb{R} \backslash\left(-r_{0}, r_{0}\right)$. For any positive real number $\delta$ and any point $\xi$ in $M$, we define the function $W_{\delta, \xi}$ on $M$ by

$$
W_{\delta, \xi}(x):=\chi\left(d_{g_{\xi}}(x, \xi)\right) \Lambda_{\xi}(x) \delta^{\frac{2-n}{2}} U\left(\delta^{-1} \exp _{\xi}^{-1}(x)\right),
$$

where $d_{g_{\xi}}$ is the geodesic distance on $M$ associated with the metric $g_{\xi}$, the exponential map is taken with respect to the same metric $g_{\xi}$ and

$$
U(x):=\left(\frac{\sqrt{n(n-2)}}{1+|x|^{2}}\right)^{\frac{n-2}{2}}
$$

for all $x \in \mathbb{R}^{n}$. For any positive real number $\delta$, the function $U_{\delta}(x)=\delta^{\frac{2-n}{2}} U\left(\delta^{-1} x\right)$ satisfies the equation $\Delta_{\text {Eucl }} U_{\delta}=U_{\delta}^{2^{\star}-1}$ where $\Delta_{\text {Eucl }}$ is the Laplace operator associated with the Euclidean metric. Moreover, by Bianchi-Egnell [4, any solution in $v \in D_{1}^{2}\left(\mathbb{R}^{n}\right)$ (the completion of $C_{c}^{\infty}\left(\mathbb{R}^{n}\right)$ for the norm $\|u\|_{D_{1}^{2}}:=\|\nabla u\|_{2}$ ) of the linear equation $\Delta_{\mathrm{Eucl}} v=\left(2^{*}-1\right) U^{2^{*}-2} v$ is a linear combination of the functions

$$
V_{0}(x):=\frac{|x|^{2}-1}{\left(1+|x|^{2}\right)^{\frac{n}{2}}} \quad \text { and } \quad V_{i}(x):=\frac{x_{i}}{\left(1+|x|^{2}\right)^{\frac{n}{2}}}
$$

for all $i=1, \ldots, n$ and $x \in \mathbb{R}^{n}$. For any positive real number $\delta$ and any point $(\xi, \omega)$ in $T M$, we define the functions $Z_{\delta, \xi}$ and $Z_{\delta, \xi, \omega}$ in $M$ by

$$
\begin{aligned}
& Z_{\delta, \xi}(x):=\chi\left(d_{g_{\xi}}(x, \xi)\right) \Lambda_{\xi}(x) \delta^{\frac{n-2}{2}} \frac{d_{g_{\xi}}(x, \xi)^{2}-\delta^{2}}{\left(\delta^{2}+d_{g_{\xi}}(x, \xi)^{2}\right)^{\frac{n}{2}}}, \\
& Z_{\delta, \xi, \omega}(x):=\chi\left(d_{g_{\xi}}(x, \xi)\right) \Lambda_{\xi}(x) \delta^{\frac{n}{2}} \frac{\left\langle\exp _{\xi}^{-1} x, \omega\right\rangle_{g_{\xi}}}{\left(\delta^{2}+d_{g_{\xi}}(x, \xi)^{2}\right)^{\frac{n}{2}}}
\end{aligned}
$$

for all $x \in M$. We then let $\Pi_{\delta, \xi}$ and $\Pi_{\delta, \xi}^{\perp}$ be the projections of the Sobolev space $H_{1}^{2}(M)$ onto the respective closed subspaces

$$
K_{\delta, \xi}:=\left\{\lambda Z_{\delta, \xi}+Z_{\delta, \xi, \omega} / \lambda \in \mathbb{R} \text { and } \omega \in T_{\xi} M\right\}
$$

and

(19)

$$
K_{\delta, \xi}^{\perp}:=\left\{\phi \in H_{1}^{2}(M) /\left\langle\phi, Z_{\delta, \xi}\right\rangle_{h}=0 \text { and }\left\langle\phi, Z_{\delta, \xi, \omega}\right\rangle_{h}=0 \quad \text { for all } \omega \in T_{\xi} M\right\},
$$

where the scalar product $\langle\cdot, \cdot\rangle_{h}$ is as in (12). 
Recall that $u_{0} \in C^{2, \theta}(M)$ is a nondegenerate positive solution to equation (77). We construct solutions of type $\left(u_{0}-B\right)$ to equations (44), or equivalently (13), like

$$
u_{\varepsilon}:=u_{0}-W_{\delta_{\varepsilon}\left(t_{\varepsilon}\right), \xi_{\varepsilon}}+\phi_{\delta_{\varepsilon}\left(t_{\varepsilon}\right), \xi_{\varepsilon}}, \quad \text { with } \delta_{\varepsilon}\left(t_{\varepsilon}\right):=t_{\varepsilon} \varepsilon^{\frac{2}{n-2}},
$$

where $W_{\delta_{\varepsilon}\left(t_{\varepsilon}\right), \xi_{\varepsilon}}$ is as in (14),$\phi_{\delta_{\varepsilon}\left(t_{\varepsilon}\right), \xi_{\varepsilon}}$ is a function in $K_{\delta_{\varepsilon}\left(t_{\varepsilon}\right), \xi_{\varepsilon}}^{\perp}$, and $t_{\varepsilon}>0$. As easily checked, if $\phi_{\delta_{\varepsilon}\left(t_{\varepsilon}\right), \xi_{\varepsilon}} \rightarrow 0$ in $H_{1}^{2}(M)$ when $\varepsilon \rightarrow 0$, then $\left(u_{\varepsilon}\right)$ is of type $u_{0}-B$. We rewrite equation (4) as the couple of equations

$$
\Pi_{\delta_{\varepsilon}(t), \xi}\left(u_{0}-W_{\delta_{\varepsilon}(t), \xi}+\phi_{\delta_{\varepsilon}(t), \xi}-i^{*}\left(f_{\varepsilon}\left(u_{0}-W_{\delta_{\varepsilon}(t), \xi}+\phi_{\delta_{\varepsilon}(t), \xi}\right)\right)\right)=0
$$

and

$$
\Pi_{\delta_{\varepsilon}(t), \xi}^{\perp}\left(u_{0}-W_{\delta_{\varepsilon}(t), \xi}+\phi_{\delta_{\varepsilon}(t), \xi}-i^{*}\left(f_{\varepsilon}\left(u_{0}-W_{\delta_{\varepsilon}(t), \xi}+\phi_{\delta_{\varepsilon}(t), \xi}\right)\right)\right)=0 .
$$

We begin with solving equation (21) in Proposition 4.1 below:

Proposition 4.1. Let $u_{0} \in C^{2, \theta}(M)$ be a positive nondegenerate solution to (7). Given two positive real numbers $a<b$, there exists a positive constant $C_{a, b}$ such that for $\varepsilon$ small, for any real number $t$ in $[a, b]$, and any point $\xi$ in $M$, there exists a unique function $\phi_{\delta_{\varepsilon}(t), \xi}$ in $K_{\delta_{\varepsilon}(t), \xi}^{\perp}$ which solves equation (21) and satisfies

$$
\left\|\phi_{\delta_{\varepsilon}(t), \xi}\right\|_{h} \leq C_{a, b} \begin{cases}\varepsilon|\ln \varepsilon| & \text { if } 3 \leq n \leq 6 \\ \varepsilon^{\frac{4}{n-2}} & \text { if } n \geq 7 \\ \varepsilon^{\frac{n+2}{2(n-2)}} & \text { if } n \geq 7, h \equiv c_{n} \operatorname{Scal}_{g}, \text { and }(M, g) \text { is loc. conf. flat. }\end{cases}
$$

Moreover, $\phi_{\delta_{\varepsilon}(t), \xi}$ is continuously differentiable with respect to $t$ and $\xi$.

The sequel of this section is devoted to the proof of Proposition 4.1. For $\varepsilon$ small, for any positive real number $\delta$, and any point $\xi$ in $M$, we let the map $L_{\varepsilon, \delta, \xi}: K_{\delta, \xi}^{\perp} \rightarrow$ $K_{\delta, \xi}^{\perp}$ be defined by

$$
L_{\varepsilon, \delta, \xi}(\phi):=\Pi_{\delta, \xi}^{\perp}\left(\phi-i^{*}\left(f_{\varepsilon}^{\prime}\left(u_{0}-W_{\delta, \xi}\right) \phi\right)\right)
$$

for all $\phi \in K_{\delta, \xi}^{\perp}$, where $u_{0} \in C^{2, \theta}(M)$ is a nondegenerate positive solution to (7), $W_{\delta, \xi}$ is as in (14) and $K_{\delta, \xi}^{\perp}$ is as in (19). Clearly, we get that $L_{\varepsilon, \delta, \xi}$ is linear and continuous. In Lemma 4.2 below, we prove the invertibility of $L_{\varepsilon, \delta, \xi}$ for $\delta$ and $\varepsilon$ small.

Lemma 4.2. Given two positive real numbers $a<b$, for $\varepsilon$ small, the map $L_{\varepsilon, \delta_{\varepsilon}(t), \xi}$ is invertible for all real numbers $t$ in $[a, b]$ and all points $\xi$ in $M$, where $\delta_{\varepsilon}(t)=$ $t \varepsilon^{2 /(n-2)}$ and $L_{\varepsilon, \delta_{\varepsilon}(t), \xi}$ is as in (23). Moreover, there exists a positive constant $C_{a, b}$ such that for $\varepsilon$ small, for any real number $t \in[a, b]$, any point $\xi \in M$, and any function $\phi \in K_{\delta_{\varepsilon}(t), \xi}^{\perp}$, there holds

$$
\left\|L_{\varepsilon, \delta_{\varepsilon}(t), \xi}(\phi)\right\|_{h} \geq C_{a, b}\|\phi\|_{h} .
$$

In particular, the inverse map $L_{\varepsilon, \delta_{\varepsilon}(t), \xi}^{-1}$ is continuous.

Proof. We prove (24). We proceed by contradiction. We assume that there exist two sequences of positive real numbers $\left(\varepsilon_{\alpha}\right)_{\alpha}$ and $\left(t_{\alpha}\right)_{\alpha}$ such that $\varepsilon_{\alpha} \rightarrow 0$ for all $\alpha \rightarrow+\infty$ and $a \leq t_{\alpha} \leq b$, a sequence of points $\left(\xi_{\alpha}\right)_{\alpha}$ in $M$, and a sequence of functions $\left(\phi_{\alpha}\right)_{\alpha}$ such that

$$
\phi_{\alpha} \in K_{\delta_{\varepsilon_{\alpha}}\left(t_{\alpha}\right), \xi_{\alpha}}^{\perp}, \quad\left\|\phi_{\alpha}\right\|_{h}=1, \quad \text { and }\left\|L_{\varepsilon_{\alpha}, \delta_{\varepsilon_{\alpha}}\left(t_{\alpha}\right), \xi_{\alpha}}\left(\phi_{\alpha}\right)\right\|_{h} \longrightarrow 0
$$


as $\alpha \rightarrow+\infty$. We define $W_{\alpha}:=W_{\delta_{\varepsilon_{\alpha}}\left(t_{\alpha}\right), \xi_{\alpha}}$. First, we claim that

$$
\left\|\phi_{\alpha}-i^{*}\left(f_{\varepsilon_{\alpha}}^{\prime}\left(u_{0}-W_{\alpha}\right) \phi_{\alpha}\right)\right\|_{h} \longrightarrow 0
$$

as $\alpha \rightarrow+\infty$. Passing if necessary to a subsequence, we may assume that all the points $\xi_{\alpha}$ belong to a small open subset $\Omega$ in $M$ on which there exists a smooth orthonormal frame. Thanks to this frame, we identify the tangent space $T_{\xi} M$ with $\mathbb{R}^{n}$ for all points $\xi$ in $\Omega$, so that $\exp _{\xi}$ is in fact the composition of the standard exponential map with a linear isometry $\Psi_{\xi}: \mathbb{R}^{n} \rightarrow T_{\xi} M$ which is smooth with respect to $\xi$. We define

$$
Z_{0, \alpha}:=Z_{\delta_{\varepsilon_{\alpha}}\left(t_{\alpha}\right), \xi_{\alpha}} \quad \text { and } \quad Z_{i, \alpha}=Z_{\delta_{\varepsilon_{\alpha}}\left(t_{\alpha}\right), \xi_{\alpha}, e_{i}}
$$

for all $i=1, \ldots, n$, where $e_{i}$ is the $i$-th vector in the canonical basis of $\mathbb{R}^{n}$ and the functions $Z_{\delta_{\varepsilon_{\alpha}}\left(t_{\alpha}\right), \xi_{\alpha}}$ and $Z_{\delta_{\varepsilon_{\alpha}}\left(t_{\alpha}\right), \xi_{\alpha}, e_{i}}$ are as in (17)-(18). For any $\alpha$, by definition of $L_{\varepsilon, \delta_{\varepsilon_{\alpha}}}\left(t_{\alpha}\right), \xi_{\alpha}$, we get that

$$
\phi_{\alpha}-i^{*}\left(f_{\varepsilon_{\alpha}}^{\prime}\left(u_{0}-W_{\alpha}\right) \phi_{\alpha}\right)-L_{\varepsilon_{\alpha}, \delta_{\varepsilon_{\alpha}}\left(t_{\alpha}\right), \xi_{\alpha}}\left(\phi_{\alpha}\right)=\sum_{i=0}^{n} \lambda_{i, \alpha} Z_{i, \alpha}
$$

for some real numbers $\lambda_{i, \alpha}$, where the functions $Z_{i, \alpha}$ are as in (27). Taking into account (25) and (28), one sees that in order to get (26), it suffices to prove that $\lambda_{i, \alpha} \rightarrow 0$ as $\alpha \rightarrow+\infty$ for all $i=0, \ldots, n$. As is easily checked, for any $i, j=0, \ldots, n$, there holds

$$
\left\langle Z_{i, \alpha}, Z_{j, \alpha}\right\rangle_{h} \longrightarrow\left\|\nabla V_{i}\right\|_{2}^{2} \delta_{i j}
$$

as $\alpha \rightarrow+\infty$ where the function $V_{i}$ is as in (16) and the real numbers $\delta_{i j}$ are the Kronecker symbols. By (28), (29), and since the functions $\phi_{\alpha}$ and $L_{\varepsilon_{\alpha}, \delta_{\varepsilon_{\alpha}}}\left(t_{\alpha}\right), \xi_{\alpha}\left(\phi_{\alpha}\right)$ belong to $K_{\delta_{\varepsilon_{\alpha}}\left(t_{\alpha}\right), \xi_{\alpha}}^{\perp}$, for any $i=0, \ldots, n$, we get that

$$
\int_{M} f_{\varepsilon_{\alpha}}^{\prime}\left(u_{0}-W_{\alpha}\right) Z_{i, \alpha} \phi_{\alpha} d v_{g}=-\lambda_{i, \alpha}\left\|\nabla V_{i}\right\|_{2}^{2}+\mathrm{o}\left(\sum_{j=0}^{n}\left|\lambda_{j, \alpha}\right|\right)
$$

as $\alpha \rightarrow+\infty$. As is easily checked, we get that

$$
\int_{M} f_{\varepsilon_{\alpha}}^{\prime}\left(u_{0}-W_{\alpha}\right) Z_{i, \alpha} \phi_{\alpha} d v_{g}=\int_{M} f_{\varepsilon_{\alpha}}^{\prime}\left(W_{\alpha}\right) Z_{i, \alpha} \phi_{\alpha} d v_{g}+\mathrm{o}(1)
$$

as $\alpha \rightarrow+\infty$. We find

$$
\frac{\int_{M} f_{\varepsilon_{\alpha}}^{\prime}\left(W_{\alpha}\right) Z_{i, \alpha} \phi_{\alpha} d v_{g}}{2^{\star}-1-\varepsilon_{\alpha}}=\delta_{\varepsilon_{\alpha}}\left(t_{\alpha}\right)^{\frac{n-2}{2} \varepsilon_{\alpha}} \int_{\mathbb{R}^{n}} \chi_{\alpha} \Lambda_{\alpha}^{2^{\star}-1-\epsilon_{\alpha}} U^{2^{*}-2-\varepsilon_{\alpha}} V_{i} \widetilde{\phi}_{\alpha} d v_{\widetilde{g}_{\alpha}}
$$

as $\alpha \rightarrow+\infty$, where the functions $U$ and $V_{i}$ are as in (15) and (16), the cutoff function $\chi$ is as in Section 4 and

$$
\begin{aligned}
& \chi_{\alpha}:=\chi\left(\delta_{\varepsilon_{\alpha}}\left(t_{\alpha}\right)|x|\right)^{2^{*}-2-\varepsilon_{\alpha}} \\
& \Lambda_{\alpha}:=\Lambda_{\xi_{\alpha}}\left(\exp _{\xi_{\alpha}}\left(\delta_{\varepsilon_{\alpha}}\left(t_{\alpha}\right) x\right)\right) \\
& \widetilde{\phi}_{\alpha}(x):=\delta_{\varepsilon_{\alpha}}\left(t_{\alpha}\right)^{\frac{n-2}{2}} \chi\left(\delta_{\varepsilon_{\alpha}}\left(t_{\alpha}\right)|x|\right) \phi_{\alpha}\left(\exp _{\xi_{\alpha}}\left(\delta_{\varepsilon_{\alpha}}\left(t_{\alpha}\right) x\right)\right), \\
& \widetilde{g}_{\alpha}(x):=\exp _{\xi_{\alpha}}^{*} g\left(\delta_{\varepsilon_{\alpha}}\left(t_{\alpha}\right) x\right)
\end{aligned}
$$

for any $\alpha \rightarrow+\infty$ and $x \in \mathbb{R}^{n}$ small enough. In the definitions above, the exponential map is taken with respect to the metric $g_{\xi_{\alpha}}$. Since $\left(\phi_{\alpha}\right)_{\alpha}$ is bounded in $H_{1}^{2}(M)$, we get that $\left(\widetilde{\phi}_{\alpha}\right)_{\alpha}$ is bounded in $D^{1,2}\left(\mathbb{R}^{n}\right)$. Passing to a subsequence, we may assume 
FRÉDÉRIC ROBERT AND JÉRÔME VÉTOIS

that $\left(\widetilde{\phi}_{\alpha}\right)_{\alpha}$ converges weakly to some function $\tilde{\phi}$ in $D^{1,2}\left(\mathbb{R}^{n}\right)$. Passing to the limit into (32) yields

$$
\int_{M} f_{\varepsilon_{\alpha}}^{\prime}\left(W_{\alpha}\right) Z_{i, \alpha} \phi_{\alpha} d v_{g} \longrightarrow\left(2^{*}-1\right) \int_{\mathbb{R}^{n}} U^{2^{*}-2} V_{i} \tilde{\phi} d x
$$

as $\alpha \rightarrow+\infty$. Since the function $V_{i}$ satisfies the equation $\Delta_{\text {Eucl }} V_{i}=\left(2^{*}-1\right) U^{2^{*}-2} V_{i}$ in $\mathbb{R}^{n}$, and since, for any $\alpha$, the function $\phi_{\alpha}$ belongs to $K_{\delta_{\varepsilon_{\alpha}}}^{\perp}\left(t_{\alpha}\right), \xi_{\alpha}$, passing to the limit as $\alpha \rightarrow+\infty$ into the equation $\left\langle Z_{i, \alpha}, \phi_{\alpha}\right\rangle_{h}=0$, we get that

$$
\int_{\mathbb{R}^{n}}\left\langle\nabla V_{i}, \nabla \tilde{\phi}\right\rangle d x=\left(2^{*}-1\right) \int_{\mathbb{R}^{n}} U^{2^{*}-2} V_{i} \tilde{\phi} d x=0 .
$$

By (30), (31), (35), and (36), we get that

$$
\lambda_{i, \alpha}=\mathrm{o}(1)+\mathrm{o}\left(\sum_{j=0}^{n}\left|\lambda_{j, \alpha}\right|\right)
$$

as $\alpha \rightarrow+\infty$. It follows that $\lambda_{i, \alpha} \rightarrow 0$ as $\alpha \rightarrow+\infty$ for all $i=0, \ldots, n$. The claim (26) then follows from (25) and (28).

For any sequence $\left(\varphi_{\alpha}\right)_{\alpha}$ in $H_{1}^{2}(M)$, and by (26), we get that

$$
\begin{aligned}
& \left|\left\langle\phi_{\alpha}, \varphi_{\alpha}\right\rangle_{h}-\int_{M} f_{\varepsilon_{\alpha}}^{\prime}\left(u_{0}-W_{\alpha}\right) \phi_{\alpha} \varphi_{\alpha} d v_{g}\right| \\
& \quad=\left|\left\langle\phi_{\alpha}-i^{*}\left(f_{\varepsilon_{\alpha}}^{\prime}\left(u_{0}-W_{\alpha}\right) \phi_{\alpha}\right), \varphi_{\alpha}\right\rangle_{h}\right| \\
& \quad \leq\left\|\phi_{\alpha}-i^{*}\left(f_{\varepsilon_{\alpha}}^{\prime}\left(u_{0}-W_{\alpha}\right) \phi_{\alpha}\right)\right\|_{h}\left\|\varphi_{\alpha}\right\|_{h}=\mathrm{o}\left(\left\|\varphi_{\alpha}\right\|_{h}\right)
\end{aligned}
$$

as $\alpha \rightarrow+\infty$.

We claim that $\phi_{\alpha} \rightarrow 0$ weakly in $H_{1}^{2}(M)$ when $\alpha \rightarrow+\infty$. We prove the claim. Since $\left(\phi_{\alpha}\right)$ is bounded in $H_{1}^{2}(M)$, up to a subsequence, there exists $\phi \in H_{1}^{2}(M)$ such that $\left(\phi_{\alpha}\right) \rightarrow \phi$ weakly in $H_{1}^{2}(M)$ when $\alpha \rightarrow+\infty$. Then for any $\varphi \in H_{1}^{2}(M)$, taking $\varphi_{\alpha} \equiv \varphi$ in (37) and letting $\alpha \rightarrow+\infty$ yields

$$
\langle\phi, \varphi\rangle_{h}=\int_{M}\left(2^{\star}-1\right) u_{0}^{2^{\star}-2} \phi \varphi d v_{g}
$$

for all $\varphi \in H_{1}^{2}(M)$, and then $\Delta_{g} \phi+h \phi=\left(2^{\star}-1\right) u_{0}^{2^{\star}-2} \phi$, which implies $\phi \equiv 0$ since $u_{0}$ is nondegenerate. This proves the claim.

We claim that $\tilde{\phi}_{\alpha} \rightarrow 0$ weakly in $D_{1}^{2}\left(\mathbb{R}^{n}\right)$ when $\alpha \rightarrow+\infty$, where $\tilde{\phi}_{\alpha}$ has been defined in (33). We prove the claim. Given a smooth function $\varphi$ with compact support in $\mathbb{R}^{n}$, we define

$$
\varphi_{\alpha}(x):=\chi\left(d_{g_{\xi_{\alpha}}}\left(x, \xi_{\alpha}\right)\right) \delta_{\varepsilon_{\alpha}}\left(t_{\alpha}\right)^{\frac{2-n}{2}} \varphi\left(\delta_{\varepsilon_{\alpha}}\left(t_{\alpha}\right)^{-1} \exp _{\xi_{\alpha}}^{-1}(x)\right)
$$

for all $x \in M$. It follows from (37) together with a change of variable that

$$
\begin{aligned}
& \int_{\mathbb{R}^{n}} \Lambda_{\alpha}^{-2}\left\langle\nabla \widetilde{\phi}_{\alpha}, \nabla \varphi\right\rangle_{\widetilde{g}_{\alpha}} d v_{\widetilde{g}_{\alpha}}+\delta_{\varepsilon_{\alpha}}\left(t_{\alpha}\right)^{2} \int_{\mathbb{R}^{n}} \Lambda_{\alpha}^{-2^{\star}} h\left(\exp _{\xi_{\alpha}}\left(\delta_{\varepsilon_{\alpha}}\left(t_{\alpha}\right) x\right)\right) \widetilde{\phi}_{\alpha} \varphi d v_{\widetilde{g}_{\alpha}} \\
& =\delta_{\varepsilon_{\alpha}}\left(t_{\alpha}\right)^{2} \int_{\mathbb{R}^{n}} \Lambda_{\alpha}^{-2^{\star}} f_{\varepsilon_{\alpha}}^{\prime}\left(u_{0, \alpha}(x)-W_{\alpha}\left(\exp _{\xi_{\alpha}}\left(\delta_{\varepsilon_{\alpha}}\left(t_{\alpha}\right) x\right)\right)\right) \widetilde{\phi}_{\alpha} \varphi d v_{\widetilde{g}_{\alpha}}+\mathrm{o}(1)
\end{aligned}
$$


as $\alpha \rightarrow+\infty$, where $u_{0, \alpha}(\cdot):=u_{0}\left(\exp _{\xi_{\alpha}}\left(\delta_{\varepsilon_{\alpha}}\left(t_{\alpha}\right) \cdot\right)\right), \widetilde{\phi}_{\alpha}$ and $\widetilde{g}_{\alpha}$ are as in (33) and (34). One easily checks that

$$
\Lambda_{\alpha}^{-2^{\star}} \delta_{\varepsilon_{\alpha}}\left(t_{\alpha}\right)^{2} f_{\varepsilon_{\alpha}}^{\prime}\left(u_{0}\left(\exp _{\xi_{\alpha}}\left(\delta_{\varepsilon_{\alpha}}\left(t_{\alpha}\right) \cdot\right)\right)-W_{\alpha}\left(\exp _{\xi_{\alpha}}\left(\delta_{\varepsilon_{\alpha}}\left(t_{\alpha}\right) \cdot\right)\right)\right)
$$

goes to $\left(2^{*}-1\right) U^{2^{*}-2}$ as $\alpha \rightarrow+\infty$ in $C_{\text {loc }}^{0}\left(\mathbb{R}^{n}\right)$. Moreover, since $\left(\widetilde{\phi}_{\alpha}\right)_{\alpha}$ converges weakly to $\tilde{\phi}$ in $D_{1}^{2}\left(\mathbb{R}^{n}\right)$, passing to the limit into (38) as $\alpha \rightarrow+\infty$ yields

$$
\int_{\mathbb{R}^{n}}\langle\nabla \tilde{\phi}, \nabla \varphi\rangle d x=\left(2^{*}-1\right) \int_{\mathbb{R}^{n}} U^{2^{*}-2} \tilde{\phi} \varphi d x .
$$

Since (39) holds for all smooth functions $\varphi$ with compact support in $\mathbb{R}^{n}$, we get that the function $\tilde{\phi}$ satisfies the equation $\Delta_{\text {Eucl }} \tilde{\phi}=\left(2^{*}-1\right) U^{2^{*}-2} \tilde{\phi}$ in $\mathbb{R}^{n}$. By Bianchi-Egnell [4], it follows that $\tilde{\phi}=\sum_{i=0}^{n} \lambda_{i} V_{i}$ for some real numbers $\lambda_{i}$. It then follows from the orthogonality condition (36) that $\tilde{\phi} \equiv 0$ is identically zero. This proves the claim.

Letting $\varphi_{\alpha}:=\phi_{\alpha}$ and using (37) together with a change of variable, we get

$$
\begin{aligned}
\left\|\phi_{\alpha}\right\|_{h}^{2} & =\left(2^{\star}-1-\varepsilon_{\alpha}\right) \int_{M}\left|u_{0}-W_{\alpha}\right|^{2^{\star}-2-\varepsilon_{\alpha}} \phi_{\alpha}^{2} d v_{g}+\mathrm{o}(1) \\
& \leq C \int_{M} \phi_{\alpha}^{2} d v_{g}+C \int_{M}\left|W_{\alpha}\right|^{2^{\star}-2-\varepsilon_{\alpha}} \phi_{\alpha}^{2} d v_{g}+\mathrm{o}(1) \\
& \leq C \int_{M} \phi_{\alpha}^{2} d v_{g}+C \int_{M}|U|^{2^{\star}-2-\varepsilon_{\alpha}} \tilde{\phi}_{\alpha}^{2} d v_{\tilde{g}_{\alpha}}+\mathrm{o}(1)
\end{aligned}
$$

as $\alpha \rightarrow+\infty$, where $\widetilde{\phi}_{\alpha}$ and $\widetilde{g}_{\alpha}$ are as in (33) and (34). Since $\phi_{\alpha} \rightarrow 0$ strongly in $L^{2}(M),\left(\widetilde{\phi}_{\alpha}^{2}\right)_{\alpha}$ is bounded in $L^{\frac{n}{n-2}}\left(\mathbb{R}^{n}\right)$ and converges almost everywhere to 0 , standard elliptic theory yields $\phi_{\alpha} \rightarrow 0$ as $\alpha \rightarrow+\infty$ in $H_{1}^{2}(M)$. This is a contradiction with (25). This ends the proof of (24).

The invertibility of $L_{\varepsilon, \delta_{\varepsilon}(t), \xi}$ follows from the Fredholm alternative. This ends the proof of Lemma 4.2

Now, we prove Proposition 4.1 by using Lemma 4.2 together with the error estimate in Section 7

Proof of Proposition 4.1. We let $a$ and $b$ be two positive real numbers such that $a<b$. For $\varepsilon$ small, for any real number $t$ in $[a, b]$, and any point $\xi$ in $M$, equation (21) is equivalent to

$$
L_{\varepsilon, \delta_{\varepsilon}(t), \xi}(\phi)=N_{\varepsilon, \delta_{\varepsilon}(t), \xi}(\phi)+R_{\varepsilon, \delta_{\varepsilon}(t), \xi},
$$

where $\delta_{\varepsilon}(t)=t \varepsilon^{2 /(n-2)}, L_{\varepsilon, \delta_{\varepsilon}(t), \xi}$ is as in (23) , and

$$
\begin{aligned}
N_{\varepsilon, \delta_{\varepsilon}(t), \xi}(\phi):= & \Pi_{\delta_{\varepsilon}(t), \xi}^{\perp}\left(i ^ { * } \left(f_{\varepsilon}\left(u_{0}-W_{\delta_{\varepsilon}(t), \xi}+\phi\right)\right.\right. \\
& \left.\left.-f_{\varepsilon}\left(u_{0}-W_{\delta_{\varepsilon}(t), \xi}\right)-f_{\varepsilon}^{\prime}\left(u_{0}-W_{\delta_{\varepsilon}(t), \xi}\right) \phi\right)\right)
\end{aligned}
$$

and

$$
R_{\varepsilon, \delta_{\varepsilon}(t), \xi}:=\Pi_{\delta_{\varepsilon}(t), \xi}^{\perp}\left(i^{*}\left(f_{\varepsilon}\left(u_{0}-W_{\delta_{\varepsilon}(t), \xi}\right)\right)-u_{0}+W_{\delta_{\varepsilon}(t), \xi}\right) .
$$

By Lemma 4.2, for $\varepsilon$ small, we get that the map $L_{\varepsilon, \delta_{\varepsilon}(t), \xi}$ is invertible for all real numbers $t$ in $[a, b]$ and all points $\xi$ in $M$. We then let the map $T_{\varepsilon, \delta_{\varepsilon}(t), \xi}: K_{\delta_{\varepsilon}(t), \xi}^{\perp} \rightarrow$ 
$K_{\delta_{\varepsilon}(t), \xi}^{\perp}$ be defined for all $\phi \in K_{\delta_{\varepsilon}(t), \xi}^{\perp}$ by

$$
T_{\varepsilon, \delta_{\varepsilon}(t), \xi}(\phi):=L_{\varepsilon, \delta_{\varepsilon}(t), \xi}^{-1}\left(N_{\varepsilon, \delta_{\varepsilon}(t), \xi}(\phi)+R_{\varepsilon, \delta_{\varepsilon}(t), \xi}\right),
$$

where $N_{\varepsilon, \delta_{\varepsilon}(t), \xi}(\phi)$ and $R_{\varepsilon, \delta_{\varepsilon}(t), \xi}$ are as in (41) and (42). For any positive real number $\Xi$, we let $B_{\varepsilon, \delta_{\varepsilon}(t), \xi}(\Xi)$ be the closed ball defined by

$$
\bar{B}_{\varepsilon, \delta_{\varepsilon}(t), \xi}(\Xi):=\left\{\phi \in K_{\delta_{\varepsilon}(t), \xi}^{\perp} /\|\phi\|_{h} \leq \Xi \nu_{\varepsilon}\right\},
$$

where $\nu_{\varepsilon}>0$ is the error obtained in Lemma 7.1 of Section 7 namely

$$
\nu_{\varepsilon}:= \begin{cases}\varepsilon|\ln \varepsilon| & \text { if } n \leq 6 \\ \varepsilon^{\frac{4}{n-2}} & \text { if } n \geq 7 \\ \varepsilon^{\frac{n+2}{2(n-2)}} & \text { if } n \geq 7, h \equiv c_{n} \operatorname{Scal}_{g}, \text { and }(M, g) \text { loc. conformally flat. }\end{cases}
$$

We fix $\theta_{0} \in\left(0, \min \left\{1,2^{\star}-2\right\}\right)$, so that $u \mapsto f_{\varepsilon}(u)$ is locally in $C^{1, \theta_{0}}$ on $H_{1}^{2}(M)$

uniformly with respect to $\varepsilon>0$ small. By Lemma 4.2 and by continuity of $i^{*}$, for $\varepsilon$ small, for any real number $t$ in $[a, b]$, any point $\xi$ in $M$, and any functions $\phi, \phi_{1}$, and $\phi_{2}$ in $H_{1}^{2}(M)$, we get that

$$
\left\|T_{\varepsilon, \delta_{\varepsilon}(t), \xi}\left(\phi_{1}\right)-T_{\varepsilon, \delta_{\varepsilon}(t), \xi}\left(\phi_{2}\right)\right\|_{h} \leq C \cdot\left(\max \left\{\left\|\phi_{1}\right\|_{h}^{\theta_{0}},\left\|\phi_{2}\right\|_{h}^{\theta_{0}}\right\}\right) \cdot\left\|\phi_{1}-\phi_{2}\right\|_{h}
$$

for some positive constant $C$ independent of $\Xi, \varepsilon, t, \xi, \phi, \phi_{1}$, and $\phi_{2}$, where $\nu_{\varepsilon}$ is as in (43). By Lemma 7.1] we have that $\left\|T_{\varepsilon, \delta_{\varepsilon}(t), \xi}(0)\right\| \leq C \nu_{\varepsilon}$. We then get that for $\Xi>0$ large enough, and then for $\varepsilon$ small, for any real number $t$ in $[a, b]$, and any point $\xi$ in $M$, then the map $T_{\varepsilon, \delta_{\varepsilon}(t), \xi}$ is a contraction map from the closed ball $\bar{B}_{\varepsilon, \delta_{\varepsilon}(t), \xi}(\Xi)$ into itself . We then get that the map $T_{\varepsilon, \delta_{\varepsilon}(t), \xi}$ admits a unique fixed point $\phi_{\delta_{\varepsilon}(t), \xi}$ in the ball $\bar{B}_{\varepsilon, \delta_{\varepsilon}(t), \xi}(\Xi)$. In other words, the function $\phi_{\delta_{\varepsilon}(t), \xi}$ is the unique solution of equation (40), or equivalently (21), which satisfies (22) with $C_{a, b}=\Xi$.

The continuous differentiability of $(t, \xi) \mapsto \phi_{\delta_{\varepsilon}(t), \xi}$ on $(a, b) \times M$ is standard. This ends the proof of Proposition 4.1.

\section{The REDUCED PROBLEM}

For $\varepsilon$ small, we introduce the functional $J_{\varepsilon}$ defined on $H_{1}^{2}(M)$ by

$$
J_{\varepsilon}(u):=\frac{1}{2} \int_{M}|\nabla u|_{g}^{2} d v_{g}+\frac{1}{2} \int_{M} h u^{2} d v_{g}-\int_{M} F_{\varepsilon}(u) d v_{g},
$$

where $F_{\varepsilon}(u):=\int_{0}^{u} f_{\varepsilon}(s) d s$. The critical points of $J_{\varepsilon}$ are the solutions of equation (13). For any positive real number $t$ and any point $\xi$ in $M$, we define

$$
\mathcal{J}_{\varepsilon}(t, \xi):=J_{\varepsilon}\left(u_{0}-W_{\delta_{\varepsilon}(t), \xi}+\phi_{\delta_{\varepsilon}(t), \xi}\right),
$$

where $W_{\delta_{\varepsilon}(t), \xi}$ is as in (14) and $\phi_{\delta_{\varepsilon}(t), \xi}$ is given by Proposition 4.1. We solve equation (20) in Proposition 5.1 below: 
Proposition 5.1. Let $u_{0} \in C^{2, \theta}(M)$ be a positive nondegenerate solution to (77). Assume that either $\left\{h \in C^{0, \theta}(M)\right.$ and $\left.3 \leq n \leq 6\right\}$ or $\left\{h \in C^{2}(M)\right.$ and $\left.3 \leq n \leq 9\right\}$ or $\left\{(M, g)\right.$ is locally conformally flat and $\left.h \equiv c_{n} \operatorname{Scal}_{g}\right\}$. Then

$$
\begin{array}{r}
\mathcal{J}_{\varepsilon}(t, \xi)=c_{1}\left(n, u_{0}\right)+c_{2}\left(n, u_{0}\right) \varepsilon+c_{3}(n) \varepsilon \ln \varepsilon+c_{4}(n) \varepsilon \ln \frac{1}{t}+c_{5}(n)\left(\varepsilon t^{\frac{n-2}{2}} u_{0}(\xi)\right. \\
\left.+\frac{n^{\frac{n-2}{4}}(n-2)^{\frac{n-6}{4}}(n-1) \omega_{n} \varepsilon^{\frac{4}{n-2}} t^{2}}{2^{n-1}(n-4) \omega_{n-1}} \cdot\left(h(\xi)-c_{n} \operatorname{Scal}_{g}(\xi)\right) \mathbf{1}_{n \geq 6}\right) \\
+o(\varepsilon)
\end{array}
$$

as $\varepsilon \rightarrow 0$, uniformly with respect to $t$ in compact subsets of $\mathbb{R}_{>0}$ and with respect to the point $\xi$ in $M, \omega_{n}$ (resp. $\omega_{n-1}$ ) is the volume of the unit n-sphere (resp. $(n-1)$-sphere $), c_{i}\left(n, u_{0}\right)(i=1,2)$ are positive constants depending only on $n$, $u_{0}$, and the manifold, $c_{i}(n)(i=3,4,5)$ depend only on $n$, and $c_{4}(n), c_{5}(n)>0$. Moreover, given two positive real numbers $a<b$, for $\varepsilon$ small, if $\left(t_{\varepsilon}, \xi_{\varepsilon}\right) \in(a, b) \times M$ is a critical point of $\mathcal{J}_{\varepsilon}$, then the function $u_{0}-W_{\delta_{\varepsilon}\left(t_{\varepsilon}\right), \xi_{\varepsilon}}+\phi_{\delta_{\varepsilon}\left(t_{\varepsilon}\right), \xi_{\varepsilon}}$ is a solution to equation (13), or equivalently (4).

This section is devoted to the proof of Proposition 5.1. We define the optimal Sobolev constant $K_{n}$ by

$$
\frac{1}{K_{n}}:=\inf _{u \in D_{1}^{2}\left(\mathbb{R}^{n}\right) \backslash\{0\}} \frac{\|\nabla u\|_{2}}{\|u\|_{2^{\star}}}=\sqrt{\frac{n(n-2) \omega_{n}^{2 / n}}{4}},
$$

where $\omega_{n}$ is the volume of the unit $n$-sphere: see Aubin [2], Talenti [35], Rodemich [31. The infimum in (46) is achieved by the function $U$ defined in (15).

Lemma 5.2. Let $u_{0} \in C^{2}(M)$ be a positive solution to (14). Assume that either $\left\{h \in C^{0, \theta}(M)\right.$ and $\left.3 \leq n \leq 6\right\}$ or $\left\{h \in C^{2}(M)\right.$ and $\left.3 \leq n \leq 9\right\}$ or $\{(M, g)$ is locally conformally flat and $\left.h \equiv c_{n} \mathrm{Scal}_{g}\right\}$. Then

$$
\begin{aligned}
J_{\varepsilon}\left(u_{0}-W_{\delta_{\varepsilon}(t), \xi}\right)= & \frac{1}{n} \int_{M} u_{0}^{2^{*}} d v_{g}+\frac{\varepsilon}{2^{*}} \int_{M} u_{0}^{2^{*}}\left(\ln u_{0}-\frac{1}{2^{*}}\right) d v_{g} \\
+\frac{K_{n}^{-n}}{n}\left(1-\beta_{n} \varepsilon-\right. & \frac{n-2}{2} \varepsilon \ln \varepsilon-\frac{(n-2)^{2}}{4} \varepsilon \ln t+\frac{2^{n} \omega_{n-1} \varepsilon t^{\frac{n-2}{2}} u_{0}(\xi)}{(n(n-2))^{\frac{n-2}{4}} \omega_{n}} \\
& \left.+\frac{2(n-1) \varepsilon^{\frac{4}{n-2}} t^{2}}{(n-2)(n-4)}\left(h(\xi)-c_{n} \operatorname{Scal}_{g}(\xi)\right) \mathbf{1}_{n \geq 6}+o(\varepsilon)\right)
\end{aligned}
$$

as $\varepsilon \rightarrow 0$, uniformly with respect to $t$ in compact subsets of $\mathbb{R}_{>0}$ and with respect to the point $\xi$ in $M$, where $\omega_{n}$ (resp. $\omega_{n-1}$ ) is the volume of the unit n-sphere (resp. $(n-1)$-sphere), $K_{n}$ is as in (46), and

$$
\beta_{n}=2^{n-3}(n-2)^{2} \frac{\omega_{n-1}}{\omega_{n}} \int_{0}^{+\infty} \frac{r^{\frac{n-2}{2}} \ln (1+r)}{(1+r)^{n}} d r+\frac{(n-2)^{2}}{4 n}(1-n \ln \sqrt{n(n-2)}) .
$$

Proof. All our estimates in this proof are uniform with respect to $t$ in compact subsets of $\mathbb{R}_{>0}$, with respect to the point $\xi$ in $M$, and with respect to $\varepsilon$ in $\left(0, \varepsilon_{0}\right)$ for some fixed positive real number $\varepsilon_{0}$. Expanding $J_{\varepsilon}\left(u_{0}-W_{\delta_{\varepsilon}(t), \xi}\right)$, using that $u_{0}$ 
is a solution to (7) and rough estimates yield

$$
\begin{aligned}
J_{\varepsilon}\left(u_{0}-W_{\delta_{\varepsilon}(t), \xi}\right)=\frac{1}{n} \int_{M} u_{0}^{2^{*}} d v_{g}+\frac{\varepsilon}{2^{*}} & \int_{M} u_{0}^{2^{*}}\left(\ln u_{0}-\frac{1}{2^{*}}\right) d v_{g} \\
& +I_{1, \varepsilon, t, \xi}+I_{2, \varepsilon, t, \xi}-I_{3, \varepsilon, t, \xi}+\mathrm{O}\left(\varepsilon^{2}\right)
\end{aligned}
$$

when $\varepsilon \rightarrow 0$ where

$$
\begin{aligned}
& I_{1, \varepsilon, t, \xi}:=\frac{1}{2} \int_{M}\left|\nabla W_{\delta_{\varepsilon}(t), \xi}\right|_{g}^{2} d v_{g}+\frac{1}{2} \int_{M} h W_{\delta_{\varepsilon}(t), \xi}^{2} d v_{g}-\frac{1}{2^{*}-\varepsilon} \int_{M} W_{\delta_{\varepsilon}(t), \xi}^{2^{*}-\varepsilon} d v_{g}, \\
& I_{2, \varepsilon, t, \xi}:=\int_{M} u_{0} W_{\delta_{\varepsilon}(t), \xi}^{2^{*}-1-\varepsilon} d v_{g} \\
& I_{3, \varepsilon, t, \xi}:=\int_{M}\left(F_{\varepsilon}\left(u_{0}-W_{\delta_{\varepsilon}(t), \xi}\right)-F_{\varepsilon}\left(u_{0}\right)-F_{\varepsilon}\left(W_{\delta_{\varepsilon}(t), \xi}\right)\right. \\
&\left.+f_{\varepsilon}\left(u_{0}\right) W_{\delta_{\varepsilon}(t), \xi}+f_{\varepsilon}\left(W_{\delta_{\varepsilon}(t), \xi}\right) u_{0}\right) d v_{g} .
\end{aligned}
$$

We estimate these terms separately.

Step 1: Estimate of $I_{1, \varepsilon, t, \xi}$ in the locally conformally case when $h \equiv c_{n} \mathrm{Scal}_{g}$. In case $h \equiv c_{n}$ Scal $_{g}$ and the manifold is locally conformally flat, the conformal change of metric $g_{\xi}=\Lambda_{\xi}^{4 /(n-2)} g$ yields

$$
\begin{aligned}
\frac{1}{2} \int_{M}\left|\nabla W_{\delta_{\varepsilon}(t), \xi}\right|_{g}^{2} d v_{g} & +\frac{1}{2} \int_{M} h W_{\delta_{\varepsilon}(t), \xi}^{2} d v_{g}-\frac{1}{2^{*}-\varepsilon} \int_{M} W_{\delta_{\varepsilon}(t), \xi}^{2^{*}-\varepsilon} d v_{g} \\
& =\frac{1}{2} \int_{M}\left|\nabla \widetilde{W}_{\delta_{\varepsilon}(t), \xi}\right|_{g_{\xi}}^{2} d v_{g_{\xi}}-\frac{1}{2^{*}-\varepsilon} \int_{M} \Lambda_{\xi}^{-\varepsilon} \widetilde{W}_{\delta_{\varepsilon}(t), \xi}^{2^{*}-\varepsilon} d v_{g_{\xi}},
\end{aligned}
$$

where $\widetilde{W}_{\delta_{\varepsilon}(t), \xi}=W_{\delta_{\varepsilon}(t), \xi} / \Lambda_{\xi}$. In this case, since the metric $g_{\xi}$ is flat in $B_{\xi}\left(r_{0}\right)$, we find

$$
\int_{M}\left|\nabla \widetilde{W}_{\delta_{\varepsilon}(t), \xi}\right|_{g_{\xi}}^{2} d v_{g_{\xi}}=\int_{\mathbb{R}^{n}}|\nabla U|^{2} d x+\mathrm{O}\left(\delta_{\varepsilon}(t)^{n-2}\right)=K_{n}^{-n}+\mathrm{O}\left(\varepsilon^{2}\right)
$$

when $\varepsilon \rightarrow 0$. Moreover, since $g_{\xi}$ is flat around $\xi$, we get that

$$
\begin{aligned}
& \frac{1}{2^{*}-\varepsilon} \int_{M} \Lambda_{\xi}^{-\varepsilon} \widetilde{W}_{\delta_{\varepsilon}(t), \xi}^{2^{*}-\varepsilon} d v_{g_{\xi}} \\
& \quad=\frac{(n(n-2))^{\frac{n-2}{4}\left(2^{*}-\varepsilon\right)}}{2^{*}-\varepsilon} \omega_{n-1} \delta_{\varepsilon}(t)^{\frac{n-2}{2} \varepsilon} \int_{0}^{\frac{r_{0}}{2 \delta_{\varepsilon}(t)}} \frac{r^{n-1} d r}{\left(1+r^{2}\right)^{\frac{n-2}{2}\left(2^{*}-\varepsilon\right)}}+\mathrm{O}\left(\delta_{\varepsilon}(t)^{n}\right) \\
& \quad+\mathrm{O}\left(\varepsilon \delta_{\varepsilon}(t)\right) \\
& \quad=\frac{n-2}{2 n} K_{n}^{-n}\left(1+\frac{2 \beta_{n}}{n-2} \varepsilon+\varepsilon \ln \varepsilon+\frac{n-2}{2} \varepsilon \ln t\right)+\mathrm{O}\left(\varepsilon^{2}|\ln \varepsilon|^{2}\right)+\mathrm{O}\left(\varepsilon \delta_{\varepsilon}(t)\right),
\end{aligned}
$$

where $K_{n}$ is as in (46), and $\beta_{n}$ is as in (48). Therefore, we get that

$$
I_{1, \varepsilon, t, \xi}=\frac{K_{n}^{-n}}{n}\left(1-\beta_{n} \varepsilon-\frac{n-2}{2} \varepsilon \ln \varepsilon-\frac{(n-2)^{2}}{4} \varepsilon \ln t\right)+o(\varepsilon)
$$

when $\varepsilon \rightarrow 0$ uniformly for all $\xi \in M$ and $t$ in a compact of $(0,+\infty)$ when $h \equiv$ $c_{n} \mathrm{Scal}_{g}$ and $(M, g)$ is locally conformally flat. 
Step 2: Estimate of $I_{1, \varepsilon, t, \xi}$ in the general case.

Cartan's expansion of the metric in geodesic normal coordinates yields for any $\alpha, \beta=1, \ldots, n$ and for $y$ close to 0 , there holds

$$
\sqrt{\left|g\left(\exp _{\xi} y\right)\right|}=1-\frac{1}{6} \sum_{\mu, \nu=1}^{n} R_{\mu \nu}(\xi) y^{\mu} y^{\nu}+P_{3}(y)+\mathrm{O}\left(|y|^{4}\right)
$$

where the function $|g|$ is the determinant of the metric, the functions $R_{\mu \nu}$ are the components of the Ricci curvature tensor in geodesic normal coordinates associated with the map $\exp _{\xi}$ and $P_{3}(y)$ is a homogenous polynomial of degree three. Using (51) together with expression of the gradient of a radially symmetrical function in geodesic normal chart, we get that

$$
\begin{aligned}
& \int_{M}\left|\nabla W_{\delta_{\varepsilon}(t), \xi}\right|_{g}^{2} d v_{g}=n^{\frac{n-2}{2}}(n-2)^{\frac{n+2}{2}} \omega_{n-1} \\
& \quad \times \int_{0}^{\frac{r_{0}}{2 \delta_{\varepsilon}(t)}} \frac{r^{n+1}}{\left(1+r^{2}\right)^{n}}\left(1-\frac{1}{6 n} \operatorname{Scal}_{g}(\xi) \delta_{\varepsilon}(t)^{2} r^{2}+\mathrm{O}\left(\delta_{\varepsilon}(t)^{4} r^{4}\right)\right) d r \\
& \quad+\mathrm{O}\left(\delta_{\varepsilon}(t)^{n-2}\right) \\
& = \begin{cases}K_{3}^{-3}+\mathrm{O}\left(\varepsilon^{2}\right) & \text { if } n=3 \\
K_{4}^{-4}\left(1+\frac{1}{4} \operatorname{Scal}_{g}(\xi) t^{2} \varepsilon^{2} \ln \varepsilon\right)+\mathrm{O}\left(\varepsilon^{2}\right) & \text { if } n=4 \\
K_{n}^{-n}\left(1-\frac{n+2}{6 n(n-4)} \operatorname{Scal}_{g}(\xi) t^{2} \varepsilon^{\frac{4}{n-2}}\right)+\mathrm{O}\left(\varepsilon^{\frac{8}{n-2}}+\varepsilon^{2}|\ln \varepsilon|\right) & \text { if } n \geq 5\end{cases}
\end{aligned}
$$

when $\varepsilon \rightarrow 0$. Taylor's expansion at $\xi$ yields on the one hand

$$
\begin{aligned}
& \frac{1}{2^{*}-\varepsilon} \int_{M} W_{\delta_{\varepsilon}(t), \xi}^{2^{*}-\varepsilon} d v_{g}=\frac{(n(n-2))^{\frac{n-2}{4}\left(2^{*}-\varepsilon\right)}}{2^{*}-\varepsilon} \omega_{n-1} \delta_{\varepsilon}(t)^{\frac{n-2}{2} \varepsilon} \\
& \quad \times \int_{0}^{\frac{r_{0}}{2 \delta_{\varepsilon}(t)}} \frac{r^{n-1}}{\left(1+r^{2}\right)^{\frac{n-2}{2}\left(2^{*}-\varepsilon\right)}}\left(1-\frac{1}{6 n} \operatorname{Scal}_{g}(\xi) \delta_{\varepsilon}(t)^{2} r^{2}\right. \\
& \left.\quad+\mathrm{O}\left(\delta_{\varepsilon}(t)^{4} r^{4}\right)\right) d r+\mathrm{O}\left(\delta_{\varepsilon}(t)^{n}\right) \\
& =\frac{n-2}{2 n} K_{n}^{-n}\left(1+\frac{2 \beta_{n}}{n-2} \varepsilon+\varepsilon \ln \varepsilon+\frac{n-2}{2} \varepsilon \ln t-\frac{1}{6(n-2)} \operatorname{Scal}_{g}(\xi) t^{2} \varepsilon^{\frac{4}{n-2}}\right) \\
& \quad+\mathrm{O}\left(\varepsilon^{\frac{8}{n-2}}+\varepsilon^{2}|\ln \varepsilon|^{2}\right)
\end{aligned}
$$

when $\varepsilon \rightarrow 0$. On the other hand,

$$
\begin{array}{rl}
\int_{M} & h W_{\delta_{\varepsilon}(t), \xi}^{2} d v_{g}=n^{\frac{n-2}{2}}(n-2)^{\frac{n-2}{2}} \delta_{\varepsilon}(t)^{2} \\
& \times \int_{B_{\frac{r_{0}}{2 \delta_{\varepsilon}(t)}}(0)} \frac{h\left(\exp _{\xi}\left(\delta_{\varepsilon}(t) x\right)\right)}{\left(1+|x|^{2}\right)^{n-2}}\left(1+\mathrm{O}\left(\delta_{\varepsilon}(t)^{2}|x|^{2}\right)\right) d x+\mathrm{O}\left(\delta_{\varepsilon}(t)^{n-2}\right)
\end{array}
$$




$$
= \begin{cases}\mathrm{O}\left(\varepsilon^{2}\right) & \text { if } n=3 \\ -\frac{3}{2} K_{4}^{-4} h(\xi) t^{2} \varepsilon^{2} \ln \varepsilon+\mathrm{O}\left(\varepsilon^{2}\right) & \text { if } n=4 \\ \frac{4(n-1)}{n(n-2)(n-4)} K_{n}^{-n} h(\xi) t^{2} \varepsilon^{\frac{4}{n-2}}+\mathrm{O}\left(R_{\varepsilon}\right) & \text { if } n \geq 5\end{cases}
$$

when $\varepsilon \rightarrow 0$, where

$$
R_{\varepsilon}:= \begin{cases}\varepsilon^{\frac{8}{n-2}}+\varepsilon^{2}|\ln \varepsilon| & \text { if } h \in C^{2}(M) \\ \varepsilon^{\frac{2(2+\theta)}{n-2}} & \text { if } h \in C^{0, \theta}(M) .\end{cases}
$$

Plugging together (52), (54), and (53) yields

$$
\begin{aligned}
I_{1, \varepsilon, t, \xi}= & \frac{K_{n}^{-n}}{n}\left(1-\beta_{n} \varepsilon-\frac{n-2}{2} \varepsilon \ln \varepsilon-\frac{(n-2)^{2}}{4} \varepsilon \ln t\right. \\
& \left.+\frac{2(n-1) \varepsilon^{\frac{4}{n-2}} t^{2}}{(n-2)(n-4)}\left(h(\xi)-c_{n} \operatorname{Scal}_{g}(\xi)\right) \mathbf{1}_{n \geq 6}\right)+\mathrm{o}(\varepsilon)+\mathrm{O}\left(R_{\varepsilon}\right)
\end{aligned}
$$

when $\varepsilon \rightarrow 0$.

Step 3: Estimate of $I_{2, \varepsilon, t, \xi}$.

$$
\begin{aligned}
\int_{M} & u_{0} W_{\delta_{\varepsilon}(t), \xi}^{2^{*}-1-\varepsilon} d v_{g}=(n(n-2))^{\frac{n-2}{4}\left(2^{*}-1-\varepsilon\right)} \omega_{n-1} u_{0}(\xi) \delta_{\varepsilon}(t)^{\frac{n-2}{2}(1+\varepsilon)} \\
& \times \int_{0}^{\frac{r_{0}}{2 \delta_{\varepsilon}(t)}} \frac{r^{n-1}}{\left(1+r^{2}\right)^{\frac{n-2}{2}\left(2^{*}-1-\varepsilon\right)}}\left(1+\mathrm{O}\left(\delta_{\varepsilon}(t)^{2} r^{2}\right)\right) d r+\mathrm{O}\left(\delta_{\varepsilon}(t)^{\frac{n+2}{2}}\right) \\
= & \frac{2^{n} \omega_{n-1} K_{n}^{-n} u_{0}(\xi) t^{\frac{n-2}{2}} \varepsilon}{n^{\frac{n+2}{4}}(n-2)^{\frac{n-2}{4}} \omega_{n}}+\mathrm{O}\left(\varepsilon^{\frac{n+2}{n-2}}|\ln \varepsilon|+\varepsilon^{2}|\ln \varepsilon|\right)
\end{aligned}
$$

when $\varepsilon \rightarrow 0$, where $K_{n}$ is as in (46), and $\beta_{n}$ is as in (48). We have used here that $\int_{\mathbb{R}^{n}} U^{2^{\star}-1} d x=\lim _{R \rightarrow+\infty} \int_{B_{R}(0)} \Delta_{\text {Eucl }} U d x$ and integrated by parts.

Step 4: Estimate of $I_{3, \varepsilon, t, \xi}$.

We have that

$$
\begin{aligned}
& \mid \int_{M}\left(F_{\varepsilon}\left(u_{0}-W_{\delta_{\varepsilon}(t), \xi}\right)-F_{\varepsilon}\left(u_{0}\right)-F_{\varepsilon}\left(W_{\delta_{\varepsilon}(t), \xi}\right)\right. \\
& \left.\quad+f_{\varepsilon}\left(u_{0}\right) W_{\delta_{\varepsilon}(t), \xi}+f_{\varepsilon}\left(W_{\delta_{\varepsilon}(t), \xi}\right) u_{0}\right) d v_{g} \mid \\
& \leq \int_{B_{\xi}\left(\sqrt{\delta_{\varepsilon}(t)}\right)}\left|F_{\varepsilon}\left(u_{0}-W_{\delta_{\varepsilon}(t), \xi}\right)-F_{\varepsilon}\left(W_{\delta_{\varepsilon}(t), \xi}\right)+f_{\varepsilon}\left(W_{\delta_{\varepsilon}(t), \xi}\right) u_{0}\right| d v_{g} \\
& \quad+\int_{M \backslash B_{\xi}\left(\sqrt{\delta_{\varepsilon}(t)}\right)}\left|F_{\varepsilon}\left(u_{0}-W_{\delta_{\varepsilon}(t), \xi}\right)-F_{\varepsilon}\left(u_{0}\right)+f_{\varepsilon}\left(u_{0}\right) W_{\delta_{\varepsilon}(t), \xi}\right| d v_{g} \\
& \quad+\int_{B_{\xi}\left(\sqrt{\delta_{\varepsilon}(t)}\right)}\left|F_{\varepsilon}\left(u_{0}\right)\right| d v_{g}+\int_{B_{\xi}\left(\sqrt{\delta_{\varepsilon}(t)}\right)}\left|f_{\varepsilon}\left(u_{0}\right) W_{\delta_{\varepsilon}(t), \xi}\right| d v_{g} \\
& \quad+\int_{M \backslash B_{\xi}\left(\sqrt{\delta_{\varepsilon}(t)}\right)}\left|F_{\varepsilon}\left(W_{\delta_{\varepsilon}(t), \xi}\right)\right| d v_{g}+\int_{M \backslash B_{\xi}\left(\sqrt{\delta_{\varepsilon}(t)}\right)}\left|f_{\varepsilon}\left(W_{\delta_{\varepsilon}(t), \xi}\right) u_{0}\right| d v_{g}
\end{aligned}
$$


As is easily checked, Taylor expansions of $F\left(u_{0}-W_{\delta_{\varepsilon}(t), \xi}\right)$ yield

$$
\begin{gathered}
\int_{B_{\xi}\left(\sqrt{\left.\delta_{\varepsilon}(t)\right)}\right.}\left|F_{\varepsilon}\left(u_{0}-W_{\delta_{\varepsilon}(t), \xi}\right)-F_{\varepsilon}\left(W_{\delta_{\varepsilon}(t), \xi}\right)+f_{\varepsilon}\left(W_{\delta_{\varepsilon}(t), \xi}\right) u_{0}\right| d v_{g} \\
=\mathrm{O}\left(\int_{B_{\xi}\left(\sqrt{\delta_{\varepsilon}(t)}\right)} u_{0}^{2} W_{\delta_{\varepsilon}(t), \xi}^{2^{*}-2-\varepsilon} d v_{g}\right), \\
\int_{M \backslash B_{\xi}\left(\sqrt{\delta_{\varepsilon}(t)}\right)}\left|F_{\varepsilon}\left(u_{0}-W_{\delta_{\varepsilon}(t), \xi}\right)-F_{\varepsilon}\left(u_{0}\right)+f_{\varepsilon}\left(u_{0}\right) W_{\delta_{\varepsilon}(t), \xi}\right| d v_{g} \\
=\mathrm{O}\left(\int_{M \backslash B_{\xi}\left(\sqrt{\delta_{\varepsilon}(t)}\right)} u_{0}^{2^{*}-2-\varepsilon} W_{\delta_{\varepsilon}(t), \xi}^{2} d v_{g}\right) .
\end{gathered}
$$

Bounding $u_{0}$ and $W_{\delta_{\varepsilon}(t), \xi}$ pointwisely roughly from above in (58) and (59) and plugging this in (57) yields

$$
I_{3, \varepsilon, t, \xi}= \begin{cases}\mathrm{O}\left(\varepsilon^{2}\right) & \text { if } n=3 \\ \mathrm{O}\left(\varepsilon^{2}|\ln \varepsilon|\right) & \text { if } n=4 \\ \mathrm{O}\left(\varepsilon^{\frac{n}{n-2}}\right) & \text { if } n \geq 5\end{cases}
$$

when $\varepsilon \rightarrow 0$.

Step 5: End of proof of Lemma 5.2.

The asymptotic expansion (47) follows from (49), (50), (55), (56) and (60).

In Lemma 5.3 below, we show that the first order terms in the asymptotic expansion of $\mathcal{J}_{\varepsilon}(t, \xi)$, defined in (44), are the same as for $J_{\varepsilon}\left(u_{0}-W_{\delta_{\varepsilon}(t), \xi}\right)$.

Lemma 5.3. Assume that either $\{3 \leq n \leq 9\}$ or $\{(M, g)$ is locally conformally flat and $\left.h \equiv c_{n} \mathrm{Scal}_{g}\right\}$. Then

$$
\mathcal{J}_{\varepsilon}(t, \xi)=J_{\varepsilon}\left(u_{0}-W_{\delta_{\varepsilon}(t), \xi}\right)+o(\varepsilon)
$$

as $\varepsilon \rightarrow 0$, uniformly with respect to $t$ in compact subsets of $\mathbb{R}_{>0}$ and with respect to the point $\xi$ in $M$.

Proof. All the estimates in this proof are uniform with respect to $t$ on compact subsets of $\mathbb{R}_{>0}$, with respect to the point $\xi$ in $M$, and with respect to $\varepsilon$ in $\left(0, \varepsilon_{0}\right)$ for some fixed positive real number $\varepsilon_{0}$. We get that

$$
\begin{aligned}
& \mathcal{J}_{\varepsilon}(t, \xi)-J_{\varepsilon}\left(u_{0}-W_{\delta_{\varepsilon}(t), \xi}\right) \\
& \quad=\left\langle u_{0}-W_{\delta_{\varepsilon}(t), \xi}-i^{*}\left(f_{\varepsilon}\left(u_{0}-W_{\delta_{\varepsilon}(t), \xi}\right)\right), \phi_{\delta_{\varepsilon}(t), \xi}\right\rangle_{h}+\mathrm{O}\left(\left\|\phi_{\delta_{\varepsilon}(t), \xi}\right\|_{h}^{2}\right)
\end{aligned}
$$

when $\varepsilon \rightarrow 0$. Proposition 4.1 and Lemma 7.1 yield

$$
\begin{aligned}
& \left\langle u_{0}-W_{\delta_{\varepsilon}(t), \xi}-i^{*}\left(f_{\varepsilon}\left(u_{0}-W_{\delta_{\varepsilon}(t), \xi}\right)\right), \phi_{\delta_{\varepsilon}(t), \xi}\right\rangle_{h}+\mathrm{O}\left(\left\|\phi_{\delta_{\varepsilon}(t), \xi}\right\|_{h}^{2}\right) \\
& = \begin{cases}\mathrm{O}\left(\varepsilon^{2}|\ln \varepsilon|^{2}\right) & \text { if } n \leq 6 \\
\mathrm{O}\left(\varepsilon^{\frac{8}{n-2}}\right) & \text { if } n \geq 7 \\
\mathrm{O}\left(\varepsilon^{\frac{n+2}{n-2}}\right) & \text { if } n \geq 7, h \equiv c_{n} \operatorname{Scal}_{g},(M, g) \text { loc. conformally flat }\end{cases}
\end{aligned}
$$

when $\varepsilon \rightarrow 0$. Finally, (61) follows from (62), and (63). 
The asymptotic expansion (45) follows from Lemmas 5.2 and 5.3. Now, we prove the second part of Proposition 5.1 .

End of proof of Proposition 5.1. Given two positive real numbers $a<b$, it remains to prove that for $\varepsilon$ small, if $\left(t_{\varepsilon}, \xi_{\varepsilon}\right) \in[a, b] \times M$ is a critical point of $\mathcal{J}_{\varepsilon}$, then the function $u_{0}-W_{\delta_{\varepsilon}\left(t_{\varepsilon}\right), \xi_{\varepsilon}}+\phi_{\delta_{\varepsilon}\left(t_{\varepsilon}\right), \xi_{\varepsilon}}$ is a solution of equation (13). In order to prove this claim, we consider a sequence of points $\left(\xi_{\alpha}\right)_{\alpha}$ in $M$ and two sequences of positive real numbers $\left(\varepsilon_{\alpha}\right)_{\alpha}$ and $\left(t_{\alpha}\right)_{\alpha}$ such that $\varepsilon_{\alpha} \rightarrow 0$ as $\alpha \rightarrow+\infty, a \leq t_{\alpha} \leq b$, and $\left(t_{\alpha}, \xi_{\alpha}\right)$ is a critical point of $\mathcal{J}_{\varepsilon_{\alpha}}$ for all $\alpha$. It is enough to show that for $\alpha$ large, the function $u_{0}-W_{\delta_{\varepsilon_{\alpha}}\left(t_{\alpha}\right), \xi_{\alpha}}+\phi_{\delta_{\varepsilon_{\alpha}}\left(t_{\alpha}\right), \xi_{\alpha}}$ is a solution of equation (13). As in the proof of Lemma 4.2, up to a subsequence, we identify the tangent space with $\mathbb{R}^{n}$ around the $\xi_{\alpha}$ 's. We define

$$
Z_{0, \delta_{\varepsilon_{\alpha}}\left(t_{\alpha}\right), \xi_{\alpha}}:=Z_{\delta_{\varepsilon_{\alpha}}\left(t_{\alpha}\right), \xi_{\alpha}} \quad \text { and } \quad Z_{i, \delta_{\varepsilon_{\alpha}}\left(t_{\alpha}\right), \xi_{\alpha}}:=Z_{\delta_{\varepsilon_{\alpha}}\left(t_{\alpha}\right), \xi_{\alpha}, e_{i}}
$$

for all $i=1, \ldots, n$, where $e_{i}$ is the $i$-th vector in the canonical basis of $\mathbb{R}^{n}$ and the functions $Z_{\delta_{\varepsilon_{\alpha}}\left(t_{\alpha}\right), \xi_{\alpha}}$ and $Z_{\delta_{\varepsilon_{\alpha}}\left(t_{\alpha}\right), \xi_{\alpha}, e_{i}}$ are as in (17) and (18). By Proposition 4.1. we get that

$$
D J_{\varepsilon_{\alpha}}\left(u_{0}-W_{\delta_{\varepsilon_{\alpha}}\left(t_{\alpha}\right), \xi_{\alpha}}+\phi_{\delta_{\varepsilon_{\alpha}}\left(t_{\alpha}\right), \xi_{\alpha}}\right)=\sum_{i=0}^{n} \lambda_{i, \alpha}\left\langle Z_{i, \delta_{\varepsilon_{\alpha}}\left(t_{\alpha}\right), \xi_{\alpha}}, \cdot\right\rangle_{h}
$$

for some real numbers $\lambda_{i, \alpha}$, where the functions $Z_{i, \delta_{\varepsilon_{\alpha}}\left(t_{\alpha}\right), \xi_{\alpha}}$ are as in (64). It follows from (65) that

$$
\frac{\partial \mathcal{J}_{\varepsilon_{\alpha}}}{\partial t}\left(t_{\alpha}, \xi_{\alpha}\right)=\sum_{i=0}^{n} \lambda_{i, \alpha}\left\langle Z_{i, \delta_{\varepsilon_{\alpha}}}\left(t_{\alpha}\right), \xi_{\alpha},\left.\frac{d}{d t}\left(-W_{\delta_{\varepsilon_{\alpha}}(t), \xi_{\alpha}}+\phi_{\delta_{\varepsilon_{\alpha}}(t), \xi_{\alpha}}\right)\right|_{t=t_{\alpha}}\right\rangle_{h}
$$

On the one hand, we find

$$
\left.\frac{d}{d t} W_{\delta_{\varepsilon_{\alpha}}(t), \xi_{\alpha}}\right|_{t=t_{\alpha}}=\frac{n^{\frac{n-2}{4}}(n-2)^{\frac{n+2}{4}}}{2 t_{\alpha}} Z_{0, \delta_{\varepsilon_{\alpha}}\left(t_{\alpha}\right), \xi_{\alpha}} .
$$

On the other hand, for any $i=0, \ldots, n$ and any $\alpha$, since the function $\phi_{\delta_{\varepsilon_{\alpha}}\left(t_{\alpha}\right), \xi_{\alpha}}$ belongs to $K_{\delta_{\varepsilon_{\alpha}}\left(t_{\alpha}\right), \xi_{\alpha}}^{\perp}$, differentiating $\left\langle Z_{i, \delta_{\varepsilon_{\alpha}}(t), \xi_{\alpha}}, \phi_{\delta_{\varepsilon_{\alpha}}(t), \xi_{\alpha}}\right\rangle_{h}=0$ with respect to $t$ yields

$$
\left\langle Z_{i, \delta_{\varepsilon_{\alpha}}\left(t_{\alpha}\right), \xi_{\alpha}},\left.\frac{d}{d t} \phi_{\delta_{\varepsilon_{\alpha}}(t), \xi_{\alpha}}\right|_{t=t_{\alpha}}\right\rangle_{h}=-\left\langle\left.\frac{d}{d t} Z_{i, \delta_{\varepsilon_{\alpha}}(t), \xi_{\alpha}}\right|_{t=t_{\alpha}}, \phi_{\delta_{\varepsilon_{\alpha}}\left(t_{\alpha}\right), \xi_{\alpha}}\right\rangle_{h}
$$

Moreover, one easily checks

$$
\left\|\left.\frac{d}{d t} Z_{i, \delta_{\varepsilon_{\alpha}}(t), \xi_{\alpha}}\right|_{t=t_{\alpha}}\right\|_{h}=\mathrm{O}(1)
$$

as $\alpha \rightarrow+\infty$. Proposition 4.1, (68), (69), (29), (66), and (67) yield

$$
\frac{\partial \mathcal{J}_{\varepsilon_{\alpha}}}{\partial t}\left(t_{\alpha}, \xi_{\alpha}\right)=-\frac{n^{\frac{n-2}{4}}(n-2)^{\frac{n+2}{4}}}{2 t_{\alpha}} \lambda_{0, \alpha}\left\|\nabla V_{0}\right\|_{2}^{2}+\mathrm{o}\left(\sum_{i=0}^{n}\left|\lambda_{i, \alpha}\right|\right)
$$


as $\alpha \rightarrow+\infty$, where the function $V_{0}$ is as in (16). For any $i=1, \ldots, n$, by (65), we get that

$$
\begin{aligned}
& \left.\frac{d}{d y_{i}} \mathcal{J}_{\varepsilon_{\alpha}}\left(t_{\alpha}, \exp _{\xi_{\alpha}} y\right)\right|_{y=0} \\
& \quad=\sum_{j=0}^{n} \lambda_{j, \alpha}\left\langle Z_{j, \delta_{\varepsilon_{\alpha}}}\left(t_{\alpha}\right), \xi_{\alpha},\left.\frac{d}{d y_{i}}\left(-W_{\delta_{\varepsilon_{\alpha}}\left(t_{\alpha}\right), \exp _{\xi_{\alpha}} y}+\phi_{\delta_{\varepsilon_{\alpha}}\left(t_{\alpha}\right), \exp _{\xi_{\alpha}} y}\right)\right|_{y=0}\right\rangle_{h},
\end{aligned}
$$

where the exponential map is taken with respect to the metric $g_{\xi_{\alpha}}$. On the one hand, direct computations yield

$$
\left.\frac{d}{d y_{i}} W_{\delta_{\varepsilon_{\alpha}}\left(t_{\alpha}\right), \exp _{\xi_{\alpha}} y}\right|_{y=0}=\frac{n^{\frac{n-2}{4}}(n-2)^{\frac{n+2}{4}}}{\delta_{\varepsilon_{\alpha}}(t)}\left(Z_{i, \delta_{\varepsilon_{\alpha}}\left(t_{\alpha}\right), \xi_{\alpha}}+R_{i, \delta_{\varepsilon_{\alpha}}\left(t_{\alpha}\right), \xi_{\alpha}}\right)
$$

where $R_{i, \delta_{\varepsilon_{\alpha}}\left(t_{\alpha}\right), \xi_{\alpha}} \rightarrow 0$ as $\alpha \rightarrow+\infty$ in $H_{1}^{2}(M)$. For any $i=1, \ldots, n, j=0, \ldots, n$, and any $\alpha$, since the function $\phi_{\delta_{\varepsilon_{\alpha}}\left(t_{\alpha}\right), \xi_{\alpha}} \in K_{\delta_{\varepsilon_{\alpha}}\left(t_{\alpha}\right), \xi_{\alpha}}^{\perp}$, differentiating the equation $\left\langle Z_{j, \delta_{\varepsilon_{\alpha}}\left(t_{\alpha}\right), \exp _{\xi_{\alpha}} y}, \phi_{\delta_{\varepsilon_{\alpha}}\left(t_{\alpha}\right), \exp _{\xi_{\alpha}} y}\right\rangle_{h}=0$ with respect to $y_{i}$ at 0 yields

$$
\left\langle Z_{j, \delta_{\varepsilon_{\alpha}}\left(t_{\alpha}\right), \xi_{\alpha}}, \frac{d}{d y_{i}} \phi_{\delta_{\varepsilon_{\alpha}}\left(t_{\alpha}\right), \exp _{\xi_{\alpha}} y}\right\rangle_{h}=-\left\langle\frac{d}{d y_{i}} Z_{j, \delta_{\varepsilon_{\alpha}}\left(t_{\alpha}\right), \exp _{\xi_{\alpha}} y}, \phi_{\delta_{\varepsilon_{\alpha}}\left(t_{\alpha}\right), \xi_{\alpha}}\right\rangle_{h} .
$$

Moreover, one easily checks

$$
\left\|\left.\frac{d}{d y_{i}} Z_{j, \delta_{\varepsilon_{\alpha}}\left(t_{\alpha}\right), \exp _{\xi_{\alpha}} y}\right|_{y=0}\right\|_{h}=\mathrm{O}\left(\frac{1}{\delta_{\varepsilon_{\alpha}}(t)}\right)
$$

as $\alpha \rightarrow+\infty$. Similarly to the derivative in the $t$-direction, we get that

$$
\left.\delta_{\varepsilon_{\alpha}}\left(t_{\alpha}\right) \frac{d}{d y_{i}} \mathcal{J}_{\varepsilon_{\alpha}}\left(t_{\alpha}, \exp _{\xi_{\alpha}} y\right)\right|_{y=0}=-n^{\frac{n-2}{4}}(n-2)^{\frac{n+2}{4}} \lambda_{i, \alpha}\left\|\nabla V_{i}\right\|_{2}+\mathrm{o}\left(\sum_{j=0}^{n}\left|\lambda_{j, \alpha}\right|\right)
$$

as $\alpha \rightarrow+\infty$, where the function $V_{i}$ is as in (16). If $\left(t_{\alpha}, \xi_{\alpha}\right)$ is a critical point of $\mathcal{J}_{\varepsilon_{\alpha}}$ for all $\alpha$, then it follows from (70) and (171) that for any $i=0, \ldots, n$, there $\lambda_{i, \alpha}=0$ for all $i=0, \ldots, n$. By (65), if follows that for $\alpha$ large, the function $u_{0}-W_{\delta_{\varepsilon_{\alpha}}}\left(t_{\alpha}\right), \xi_{\alpha}+\phi_{\delta_{\varepsilon_{\alpha}}\left(t_{\alpha}\right), \xi_{\alpha}}$ is a critical point of the functional $J_{\varepsilon_{\alpha}}$, and therefore a solution of equation (13). This ends the proof of Proposition 5.1.

\section{Proof of the theorems}

Proof of Theorems 1.2 and 2.2. We let $\mathcal{G}$ be the function defined on $\mathbb{R}_{>0} \times M$ by

$$
\mathcal{G}(t, \xi):=c_{4}(n) \ln \frac{1}{t}+c_{5}(n) t^{\frac{n-2}{2}} u_{0}(\xi)
$$

where $c_{4}(n)$ and $c_{5}(n)$ are as in (45). Since $u_{0}$ is positive and $M$ is compact, we get

$$
\lim _{t \rightarrow 0} \mathcal{G}(t, \xi)=+\infty \quad \text { and } \quad \lim _{t \rightarrow+\infty} \mathcal{G}(t, \xi)=+\infty
$$

uniformly with respect to $\xi \in M$. Since $h \equiv c_{n} \operatorname{Scal}_{g}$ and either $\{3 \leq n \leq 9\}$ or $\{(M, g)$ is locally conformally flat $\}$, it follows from Proposition 5.1 that

$$
\lim _{\varepsilon \rightarrow 0} \frac{1}{\varepsilon}\left(\mathcal{J}_{\varepsilon}(t, \xi)-c_{1}\left(n, u_{0}\right)-c_{2}\left(n, u_{0}\right) \varepsilon-c_{3}(n) \varepsilon \ln \varepsilon\right)=\mathcal{G}(t, \xi)
$$


uniformly with respect to $t$ in compact subsets of $\mathbb{R}_{>0}$ and with respect to the point $\xi$ in $M$. For $\varepsilon$ small, by (73), (74), and by continuity of $\mathcal{J}_{\varepsilon}$ and $\mathcal{G}$, we get the existence of a family of points $\left(t_{\varepsilon}, \xi_{\varepsilon}\right)$ which realize the minimum values of the functions $\mathcal{J}_{\varepsilon}$ in $(a, b) \times M$ for some positive real numbers $a<b$ independent of $\varepsilon$. By Proposition 5.1. it follows that for $\varepsilon$ small, the function $u_{\varepsilon}=u_{0}-W_{\delta_{\varepsilon}\left(t_{\varepsilon}\right), \xi_{\varepsilon}}+\phi_{\delta_{\varepsilon}\left(t_{\varepsilon}\right), \xi_{\varepsilon}}$ is a solution of equation (2), where $W_{\delta_{\varepsilon}(t), \xi}$ is as in (14) and $\phi_{\delta_{\varepsilon}(t), \xi}$ is given by Proposition 4.1 We get that $\lim _{\varepsilon \rightarrow 0} u_{\varepsilon}=u_{0}$ in $H_{1, l o c .}^{2}\left(M \backslash\left\{\xi_{0}\right\}\right)$ where $\xi_{0}:=\lim _{\varepsilon \rightarrow 0} \xi_{\varepsilon}$ (up to a subsequence): it then follows from standard elliptic theory that $\lim _{\varepsilon \rightarrow 0} u_{\varepsilon}=u_{0}$ in $C_{\text {loc }}^{2}\left(M \backslash\left\{\xi_{0}\right\}\right)$. Independently, $\lim _{\varepsilon \rightarrow 0} \delta_{\varepsilon}\left(t_{\varepsilon}\right)^{\frac{n-2}{2}} u_{\varepsilon}\left(\exp _{\xi_{\epsilon}} \cdot\right)=-U$ in $H_{1, l o c .}^{2}\left(\mathbb{R}^{n}\right)$, and still by elliptic theory, one then gets the convergence in $C_{l o c}^{2}\left(\mathbb{R}^{n}\right)$. This proves that $\left(u_{\varepsilon}\right)_{\varepsilon>0}$ changes sign and blows-up when $\varepsilon \rightarrow 0$. This ends the proof of Theorems 1.2 and 2.2

Proof of Theorems 1.1 and 2.3. In dimensions $3 \leq n \leq 5$, the proof of Theorem 1.1 is similar to the proof of Theorem 1.2. The specificity of dimension $n=6$, is that the function $\mathcal{G}$ in (72) is replaced by

$$
\mathcal{G}(t, \xi):=c_{4}(6) \ln \frac{1}{t}+c_{5}(6)\left(u_{0}(\xi)+\frac{1}{2}\left(h(\xi)-c_{6} \operatorname{Scal}_{g}(\xi)\right)\right) t^{2},
$$

where $c_{4}(6), c_{5}(6)>0$ are as in (45): therefore (73) holds with the hypothesis of Theorem 1.1 and the proof of Theorem 1.1 goes as for Theorem 1.2. We focus on Theorem 2.3. In dimension $n=6$, computations similar to (45) yield

$$
\begin{aligned}
J_{\varepsilon}^{+}\left(u_{0}+\right. & \left.W_{\delta_{\varepsilon}(t), \xi}\right)=c_{1}\left(6, u_{0}\right)+c_{2}\left(6, u_{0}\right) \varepsilon+c_{3}(6) \varepsilon \ln \varepsilon \\
& +\left(c_{4}(6) \ln \frac{1}{t}+c_{5}(6)\left(\frac{1}{2}\left(h(\xi)-c_{6} \operatorname{Scal}_{g}(\xi)\right)-u_{0}(\xi)\right) t^{2}\right) \varepsilon+o(\varepsilon)
\end{aligned}
$$

as $\varepsilon \rightarrow 0$, where $J_{\varepsilon}^{+}(u):=\frac{1}{2} \int_{M}|\nabla u|_{g}^{2} d v_{g}+\frac{1}{2} \int_{M} h u^{2} d v_{g}-\frac{1}{2^{\star}-\varepsilon} \int_{M} u_{+}^{2^{\star}-\varepsilon} d v_{g}$. The proof then is similar to the proof of Theorem 1.1 .

Proof of Theorem [2.1. The introduction of another type of model for blow-up is required here. It follows from Lee-Parker 21] that for any $\xi \in M$, there exists $\Lambda_{\xi} \in$ $C^{\infty}(M)$ positive such that $g_{\xi}:=\Lambda_{\xi}^{\frac{4}{n-2}} g$ satisfies $d v_{g_{\xi}}=\left(1+O\left(d_{g_{\xi}}(\xi, \cdot)^{n}\right)\right) d x$ in a geodesic normal chart. An immediate consequence is that $\operatorname{Scal}_{g_{\xi}}(\xi)=\left|\nabla \operatorname{Scal}_{g_{\xi}}(\xi)\right|=$ 0 and $\Delta_{g_{\xi}} \operatorname{Scal}_{g_{\xi}}(\xi)=\frac{1}{6} \mid$ Weyl $\left._{g}(\xi)\right|_{g} ^{2}$. Moreover, we can assume that $(\xi, x) \mapsto \Lambda_{\xi}(x)$ is $C^{\infty}$ and $\nabla \Lambda_{\xi}(\xi)=0$. We define $W_{\delta, \xi}$ in (14) with the function $\Lambda_{\xi}$ above. When $h \equiv c_{n} \mathrm{Scal}_{g}$, the conformal law of change of metric yields the Taylor expansion

$$
\begin{gathered}
J_{\varepsilon}\left(u_{0}-W_{\delta_{\varepsilon}(t), \xi}\right)=c_{1}\left(n, u_{0}\right)+c_{2}\left(n, u_{0}\right) \varepsilon+c_{3}(n) \varepsilon \ln \varepsilon+\frac{K_{n}^{-n}}{n}\left(\frac{(n-2)^{2}}{4} \varepsilon \ln \frac{1}{t}\right. \\
\left.+\frac{2^{n} \omega_{n-1}}{\omega_{n}(n(n-2))^{(n-2) / 4}} u(\xi) \varepsilon t^{\frac{n-2}{2}}-\frac{\left|\mathrm{Weyl}_{g}(\xi)\right|_{g}^{2}}{24(n-4)(n-6)} \varepsilon^{\frac{8}{n-2}} t^{4}\right)+\mathrm{o}\left(\varepsilon+\varepsilon^{\frac{8}{n-2}}\right)
\end{gathered}
$$

when $\varepsilon \rightarrow 0$ for $n \geq 7$. When $n<10$, the term involving the Weyl tensor is neglictible. When $n=10$, it competes with the one involving $u_{0}$ : arguing as in the proofs above, we get the existence of a blowing-up family when $u_{0}>\frac{5}{567} \mid$ Weyl $\left._{g}\right|_{g} ^{2}$, which proves Theorem 2.1 since the additional terms involving $\phi_{\delta_{\varepsilon}(t), \xi}$ are neglictible 
when $n \leq 17$. When $n>10$, the Weyl tensor dominates but the negative sign does not allow to construct a critical point for the reduced functional.

Proof of Theorem 2.4. If $\xi_{0} \in M$ is a strict minimizer of $\Phi$ on $\bar{B}_{\xi_{0}}\left(\nu_{0}\right) \subset M$ with $\nu_{0}>0$, the arguments above extend by minimizing $\mathcal{G}$ on $(0,+\infty) \times B_{\xi_{0}}\left(\nu_{0}\right)$.

\section{ERror estimate}

This section is devoted to the error estimate used in previous sections. All notations refer to Section 4 The estimate is as follows:

Lemma 7.1. Given two positive real numbers $a<b$, there exists a positive constant $C_{a, b}^{\prime}$ such that for $\varepsilon$ small, for any real number $t$ in $[a, b]$, and any point $\xi$ in $M$, there holds

$$
\begin{aligned}
& \left\|i^{*}\left(f_{\varepsilon}\left(u_{0}-W_{\delta_{\varepsilon}(t), \xi}\right)\right)-u_{0}+W_{\delta_{\varepsilon}(t), \xi}\right\|_{h} \\
& \leq C_{a, b}^{\prime} \begin{cases}\varepsilon|\ln \varepsilon| & \text { if } n \leq 6 \\
\varepsilon^{\frac{4}{n-2}} & \text { if } n \geq 7 \\
\varepsilon^{\frac{n+2}{2(n-2)}} & \text { if } n \geq 7, h \equiv c_{n} \operatorname{Scal}_{g}, \text { and }(M, g) \text { loc. conformally flat, }\end{cases}
\end{aligned}
$$

where $\delta_{\varepsilon}(t)=t \varepsilon^{2 /(n-2)}$ and $W_{\delta_{\varepsilon}(t), \xi}$ is as in (14).

Proof. All our estimates in this proof are uniform with respect to $t$ in $[a, b], \xi$ in $M$, and $\varepsilon$ in $\left(0, \varepsilon_{0}\right)$ for some fixed positive real number $\varepsilon_{0}$. The continuity of $i^{*}$ yields

$$
\begin{aligned}
& \left\|i^{*}\left(f_{\varepsilon}\left(u_{0}-W_{\delta_{\varepsilon}(t), \xi}\right)\right)-u_{0}+W_{\delta_{\varepsilon}(t), \xi}\right\|_{h} \\
& =\mathrm{O}\left(\left\|f_{\varepsilon}\left(u_{0}-W_{\delta_{\varepsilon}(t), \xi}\right)-\left(\Delta_{g}+h\right)\left(u_{0}-W_{\delta_{\varepsilon}(t), \xi}\right)\right\|_{\frac{2 n}{n+2}}\right) .
\end{aligned}
$$

It follows that

$$
\left\|i^{*}\left(f_{\varepsilon}\left(u_{0}-W_{\delta_{\varepsilon}(t), \xi}\right)\right)-u_{0}+W_{\delta_{\varepsilon}(t), \xi}\right\|_{h}=\mathrm{O}\left(\tilde{I}_{1, \varepsilon, t, \xi}+\tilde{I}_{2, \varepsilon, t, \xi}+\tilde{I}_{3, \varepsilon, t, \xi}\right),
$$

where

$$
\begin{gathered}
\tilde{I}_{1, \varepsilon, t, \xi}:=\left\|f_{\varepsilon}\left(u_{0}-W_{\delta_{\varepsilon}(t), \xi}\right)-f_{\varepsilon}\left(u_{0}\right)+f_{\varepsilon}\left(W_{\delta_{\varepsilon}(t), \xi}\right)\right\|_{\frac{2 n}{n+2}}, \\
\tilde{I}_{2, \varepsilon, t, \xi}:=\left\|f_{\varepsilon}\left(u_{0}\right)-\Delta_{g} u_{0}-h u_{0}\right\|_{\frac{2 n}{n+2}}, \\
\tilde{I}_{3, \varepsilon, t, \xi}:=\left\|f_{\varepsilon}\left(W_{\delta_{\varepsilon}(t), \xi}\right)-\Delta_{g} W_{\delta_{\varepsilon}(t), \xi}-h W_{\delta_{\varepsilon}(t), \xi}\right\|_{\frac{2 n}{n+2}} .
\end{gathered}
$$

We estimate these terms separately.

Step 1: Estimate of $\tilde{I}_{1, \varepsilon, t, \xi}$.

We get

$$
\begin{aligned}
\tilde{I}_{1, \varepsilon, t, \xi} \leq \|\left(f_{\varepsilon}\right. & \left.\left(u_{0}-W_{\delta_{\varepsilon}(t), \xi}\right)+f_{\varepsilon}\left(W_{\delta_{\varepsilon}(t), \xi}\right)\right) \mathbf{1}_{B_{\xi}\left(\sqrt{\delta_{\varepsilon}(t)}\right)} \|_{\frac{2 n}{n+2}} \\
+ & \left\|\left(f_{\varepsilon}\left(u_{0}-W_{\delta_{\varepsilon}(t), \xi}\right)-f_{\varepsilon}\left(u_{0}\right)\right) \mathbf{1}_{M \backslash B_{\xi}\left(\sqrt{\delta_{\varepsilon}(t)}\right)}\right\|_{\frac{2 n}{n+2}} \\
& +\left\|f_{\varepsilon}\left(W_{\delta_{\varepsilon}(t), \xi}\right) \mathbf{1}_{M \backslash B_{\xi}\left(\sqrt{\delta_{\varepsilon}(t)}\right)}\right\|_{\frac{2 n}{n+2}}+\left\|f_{\varepsilon}\left(u_{0}\right) \mathbf{1}_{B_{\xi}\left(\sqrt{\delta_{\varepsilon}(t)}\right)}\right\|_{\frac{2 n}{n+2}} .
\end{aligned}
$$


FRÉDÉRIC ROBERT AND JÉRÔME VÉTOIS

As is easily checked, Taylor's expansion for $f_{\varepsilon}\left(u_{0}-W_{\delta_{\varepsilon}(t), \xi}\right)$ yields

$$
\begin{aligned}
& \left\|\left(f_{\varepsilon}\left(u_{0}-W_{\delta_{\varepsilon}(t), \xi}\right)+f_{\varepsilon}\left(W_{\delta_{\varepsilon}(t), \xi}\right)\right) \mathbf{1}_{B_{\xi}\left(\sqrt{\delta_{\varepsilon}(t)}\right)}\right\|_{\frac{2 n}{n+2}} \\
& \leq C\left(\left\|u_{0} W_{\delta_{\varepsilon}(t), \xi}^{2^{*}-2-\varepsilon} \mathbf{1}_{B_{\xi}\left(\sqrt{\delta_{\varepsilon}(t)}\right)}\right\|_{\frac{2 n}{n+2}}+\left\|u_{0}^{2^{*}-1-\varepsilon} \mathbf{1}_{B_{\xi}\left(\sqrt{\delta_{\varepsilon}(t)}\right)}\right\|_{\frac{2 n}{n+2}}\right)
\end{aligned}
$$

and

$$
\begin{aligned}
& \left\|\left(f_{\varepsilon}\left(u_{0}-W_{\delta_{\varepsilon}(t), \xi}\right)-f_{\varepsilon}\left(u_{0}\right)\right) \mathbf{1}_{M \backslash B_{\xi}\left(\sqrt{\delta_{\varepsilon}(t)}\right)}\right\|_{\frac{2 n}{n+2}} \\
& \quad \leq C\left(\left\|u_{0}^{2^{*}-2-\varepsilon} W_{\delta_{\varepsilon}(t), \xi} \mathbf{1}_{M \backslash B_{\xi}\left(\sqrt{\delta_{\varepsilon}(t)}\right)}\right\|_{\frac{2 n}{n+2}}+\left\|W_{\delta_{\varepsilon}(t), \xi}^{2^{*}-1-\varepsilon} \mathbf{1}_{M \backslash B_{\xi}\left(\sqrt{\delta_{\varepsilon}(t)}\right)}\right\|_{\frac{2 n}{n+2}}\right) .
\end{aligned}
$$

Estimating roughly these terms yields

$$
\tilde{I}_{1, \varepsilon, t, \xi}= \begin{cases}\mathrm{O}(\varepsilon) & \text { if } n \leq 5 \\ \mathrm{O}\left(\varepsilon|\ln \varepsilon|^{\frac{2}{3}}\right) & \text { if } n=6 \\ \mathrm{O}\left(\varepsilon^{\frac{n+2}{2(n-2)}}\right) & \text { if } n \geq 7\end{cases}
$$

when $\varepsilon \rightarrow 0$.

Step 2: Estimate of $\tilde{I}_{2, \varepsilon, t, \xi}$.

Since $u_{0}$ is a solution of (7), we get that

$$
\tilde{I}_{2, \varepsilon, t, \xi}=\left\|f_{\varepsilon}\left(u_{0}\right)-f_{0}\left(u_{0}\right)\right\|_{\frac{2 n}{n+2}}=\mathrm{O}(\varepsilon) .
$$

Step 3: Estimate of $\tilde{I}_{3, \varepsilon, t, \xi}$.

We define $\chi_{\xi}(\cdot)=\chi\left(d_{g_{\xi}}(\cdot, \xi)\right), U_{\delta, \xi}(\cdot)=\delta^{\frac{2-n}{2}} U\left(\delta^{-1} \exp _{\xi}^{-1}(\cdot)\right)$, where the function $U$ is as in (15) and the exponential map is taken with respect to the metric $g_{\xi}$.

Step 3.1: Estimate of $I_{3, \varepsilon, t, \xi}$ when $(M, g)$ is locally conformally flat and $h \equiv$ $c_{n} \mathrm{Scal}_{g}$.

Since $g_{\xi}=\Lambda_{\xi}^{4 /(n-2)} g$ is flat, we get that

$$
f_{\varepsilon}\left(W_{\delta_{\varepsilon}(t), \xi}\right)-\Delta_{g} W_{\delta_{\varepsilon}(t), \xi}-h W_{\delta_{\varepsilon}(t), \xi}=\Lambda_{\xi}^{2^{*}-1}\left(\Lambda_{\xi}^{-\varepsilon} f_{\varepsilon}\left(\widetilde{W}_{\delta_{\varepsilon}(t), \xi}\right)-\Delta_{g_{\xi}} \widetilde{W}_{\delta_{\varepsilon}(t), \xi}\right)
$$

where $\widetilde{W}_{\delta_{\varepsilon}(t), \xi}=W_{\delta_{\varepsilon}(t), \xi} / \Lambda_{\xi}$. In this case, since the metric $g_{\xi}$ is flat in $B_{\xi}\left(r_{0}\right)$ and since the function $U$ is a solution of the equation $\Delta_{\mathrm{Eucl}} U=U^{2^{*}-1}$ in $\mathbb{R}^{n}$, we get that

$$
\begin{aligned}
& I_{3, \varepsilon, t, \xi} \leq\left\|\left(\chi_{\xi} \Lambda_{\xi}\right)^{2^{*}-1-\varepsilon}\left(U_{\delta_{\varepsilon}(t), \xi}^{2^{*}-1-\varepsilon}-U_{\delta_{\varepsilon}(t), \xi}^{2^{*}-1}\right)\right\|_{\frac{2 n}{n+2}} \\
& +\left\|\left(\chi_{\xi}^{2^{*}-1-\varepsilon} \Lambda_{\xi}^{-\varepsilon}-\chi_{\xi}\right) \Lambda_{\xi}^{2^{*}-1} U_{\delta_{\varepsilon}(t), \xi}^{2^{*}-1}\right\|_{\frac{2 n}{n+2}}+\left\|\Lambda_{\xi}^{2^{\star}-1} U_{\delta_{\varepsilon}(t), \xi} \Delta_{g_{\xi}} \chi_{\xi}\right\|_{\frac{2 n}{n+2}} \\
& +2\left\|\Lambda_{\xi}^{2^{\star}-1}\left\langle\nabla \chi_{\xi}, \nabla U_{\delta_{\varepsilon}(t), \xi}\right\rangle_{g_{\xi}}\right\|_{\frac{2 n}{n+2}}
\end{aligned}
$$


Step 3.2: Estimate of $I_{3, \varepsilon, t, \xi}$ in the general case.

In general, we get that

$$
\begin{gathered}
I_{3, \varepsilon, t, \xi} \leq\left\|\chi_{\xi}^{2^{*}-1-\varepsilon}\left(U_{\delta_{\varepsilon}(t), \xi}^{2^{*}-1-\varepsilon}-U_{\delta_{\varepsilon}(t), \xi}^{2^{*}-1}\right)\right\|_{\frac{2 n}{n+2}}+\left\|\left(\chi_{\xi}^{2^{*}-1-\varepsilon}-\chi_{\xi}\right) U_{\delta_{\varepsilon}(t), \xi}^{2^{*}-1}\right\|_{\frac{2 n}{n+2}} \\
+\left\|\chi_{\xi}\left(U_{\delta_{\varepsilon}(t), \xi}^{2^{*}-1}-\Delta_{g} U_{\delta_{\varepsilon}(t), \xi}\right)\right\|_{\frac{2 n}{n+2}}+\left\|U_{\delta_{\varepsilon}(t), \xi} \Delta_{g} \chi_{\xi}\right\|_{\frac{2 n}{n+2}} \\
+2\left\|\left\langle\nabla \chi_{\xi}, \nabla U_{\delta_{\varepsilon}(t), \xi}\right\rangle_{g}\right\|_{\frac{2 n}{n+2}}+\left\|h \chi_{\xi} U_{\delta_{\varepsilon}(t), \xi}\right\|_{\frac{2 n}{n+2}} .
\end{gathered}
$$

Step 3.3: Estimates of the terms in (80) and (81).

Since $\chi_{\xi} \equiv 1$ on $B_{\xi}\left(r_{0} / 2\right)$ and $\chi_{\xi} \equiv 0$ on $M \backslash B_{\xi}\left(r_{0}\right)$, we get that

$$
\begin{aligned}
& \int_{M}\left|\left(\chi_{\xi} \Lambda_{\xi}\right)^{2^{*}-1-\varepsilon}\left(U_{\delta_{\varepsilon}(t), \xi}^{2^{*}-1-\varepsilon}-U_{\delta_{\varepsilon}(t), \xi}^{2^{*}-1}\right)\right|^{\frac{2 n}{n+2}} d v_{g}=\mathrm{O}\left(\varepsilon^{\frac{2 n}{n+2}}|\ln \varepsilon|^{\frac{2 n}{n+2}}\right), \\
& \int_{M}\left|\left(\chi_{\xi}^{2^{*}-1-\varepsilon} \Lambda_{\xi}^{-\varepsilon}-\chi_{\xi}\right) \Lambda_{\xi}^{2^{*}-1} U_{\delta_{\varepsilon}(t), \xi}^{2^{*}-1}\right|^{\frac{2 n}{n+2}} d v_{g}=\mathrm{O}\left(\varepsilon^{\frac{2 n}{n+2}}\right), \\
& \int_{M}\left|\Lambda_{\xi}^{2^{\star}-1} U_{\delta_{\varepsilon}(t), \xi} \Delta_{g} \chi_{\xi}\right|^{\frac{2 n}{n+2}}=\mathrm{O}\left(\varepsilon^{\frac{2 n}{n+2}}\right)
\end{aligned}
$$

when $\varepsilon \rightarrow 0$. A rough $L^{\infty}$ upper bound for $\left|\nabla U_{\delta_{\varepsilon}(t), \xi}\right|$ on $M \backslash B_{r_{0} / 2}(\xi)$ yields

$$
\int_{M}\left|\left\langle\Lambda_{\xi}^{2^{\star}-1} \nabla \chi_{\xi}, \nabla U_{\delta_{\varepsilon}(t), \xi}\right\rangle_{g}\right|^{\frac{2 n}{n+2}} d v_{g}=\mathrm{O}\left(\varepsilon^{\frac{2 n}{n+2}}\right) .
$$

Since $\Delta_{\text {Eucl }} U=U^{2^{\star}-1}$, we get in the chart $\exp _{\xi}$ that

$$
\Delta_{g} U_{\delta_{\varepsilon}(t), \xi}-U_{\delta_{\varepsilon}(t), \xi}^{2^{\star}-1}=-\left(g^{i j}-\delta^{i j}\right) \partial_{i j} U_{\delta_{\varepsilon}(t), \xi}+g^{i j} \Gamma_{i j}^{k} \partial_{k} U_{\delta_{\varepsilon}(t), \xi},
$$

where the $g^{i j}$ 's are the coordinate of the metric $g=g_{\xi}$ and $\Gamma_{\alpha \beta}^{\gamma}$ 's are the Christoffel symbols of the metric $g$ in the normal chart $\exp _{\xi}$. Cartan's expansion of the metric yields $\left|g^{i j}(x)-\delta^{i j}\right| \leq C|x|^{2}$ and $\left|\Gamma_{i j}^{k}(x)\right| \leq C|x|$ around 0 , and therefore

$$
\left|U_{\delta_{\varepsilon}(t), \xi}^{2^{\star}-1}-\Delta_{g} U_{\delta_{\varepsilon}(t), \xi}\right| \leq C|x|^{2}\left|\nabla^{2} U_{\delta_{\varepsilon}(t), \xi}\right|+C|x| \cdot\left|\nabla U_{\delta_{\varepsilon}(t), \xi}\right|
$$

via the chart $\exp _{\xi}$. Therefore, we get that

$$
\int_{M}\left|\chi_{\xi}\left(U_{\delta_{\varepsilon}(t), \xi}^{2^{\star}-1}-\Delta_{g} U_{\delta_{\varepsilon}(t), \xi}\right)\right|^{\frac{2 n}{n+2}} d v_{g}= \begin{cases}\mathrm{O}\left(\varepsilon^{\frac{2 n}{n+2}}\right) & \text { if } n \leq 5 \\ \mathrm{O}\left(\varepsilon^{\frac{3}{2}}|\ln \varepsilon|\right) & \text { if } n=6 \\ \mathrm{O}\left(\varepsilon^{\frac{8 n}{(n+2)(n-2)}}\right) & \text { if } n \geq 7 .\end{cases}
$$

It remains to compute

$$
\int_{M}\left|h \chi_{\xi} U_{\delta_{\varepsilon}(t), \xi}\right|^{\frac{2 n}{n+2}} d v_{g}= \begin{cases}\mathrm{O}\left(\varepsilon^{\frac{2 n}{n+2}}\right) & \text { if } n \leq 5 \\ \mathrm{O}\left(\varepsilon^{\frac{3}{2}}|\ln \varepsilon|\right) & \text { if } n=6 \\ \mathrm{O}\left(\varepsilon^{\frac{8 n}{(n+2)(n-2)}}\right) & \text { if } n \geq 7\end{cases}
$$

when $\varepsilon \rightarrow 0$. 
Step 3.4: End of estimate of $\tilde{I}_{3, \varepsilon, t, \xi}$.

By (81)-(87), we get

$$
\left\|f_{\varepsilon}\left(W_{\delta_{\varepsilon}(t), \xi}\right)-\Delta_{g} W_{\delta_{\varepsilon}(t), \xi}-h W_{\delta_{\varepsilon}(t), \xi}\right\|_{\frac{2 n}{n+2}}= \begin{cases}\mathrm{O}(\varepsilon|\ln \varepsilon|) & \text { if } n \leq 6 \\ \mathrm{O}\left(\varepsilon^{\frac{4}{n-2}}\right) & \text { if } n \geq 7 .\end{cases}
$$

In case $h \equiv c_{n}$ Scal $_{g}$ and the manifold is locally conformally flat, by (80), (82)-(85), we get

$$
\left\|f_{\varepsilon}\left(W_{\delta_{\varepsilon}(t), \xi}\right)-\Delta_{g} W_{\delta_{\varepsilon}(t), \xi}-h W_{\delta_{\varepsilon}(t), \xi}\right\|_{\frac{2 n}{n+2}}=\mathrm{O}(\varepsilon|\ln \varepsilon|)
$$

when $\varepsilon \rightarrow 0$.

Step 4: End of proof of (76).

Finally, (76) follows from (77), (78), (79), (88), and (89).

\section{REFERENCES}

[1] A. Ambrosetti and A. Malchiodi, Perturbation methods and semilinear elliptic problems on $\mathbb{R}^{n}$, Progress in Mathematics, vol. 240, Birkhäuser Verlag, Basel, 2006.

[2] Th. Aubin, Problèmes isopérimétriques et espaces de Sobolev, J. Differential Geometry 11 (1976), no. 4, 573-598.

[3] M. Berger, P. Gauduchon, and E. Mazet, Le spectre d'une variété riemannienne, Lecture Notes in Mathematics, Vol. 194, Springer-Verlag, Berlin, 1971.

[4] G. Bianchi and H. Egnell, A note on the Sobolev inequality, J. Funct. Anal. 100 (1991), no. 1, $18-24$.

[5] S. Brendle, Blow-up phenomena for the Yamabe equation, J. Amer. Math. Soc. 21 (2008), no. 4, 951-979.

[6] S. Brendle and F. C. Marques, Blow-up phenomena for the Yamabe equation. II, J. Differential Geom. 81 (2009), no. 2, 225-250.

[7] W. Chen, J. Wei, and S. Yan, Infinitely many solutions for the Schrödinger equations in $\mathbb{R}^{N}$ with critical growth, J. Differential Equations. To appear.

[8] M. del Pino, M. Musso, F. Pacard, and A. Pistoia, Large energy entire solutions for the Yamabe equation, J. Differential Equations 251 (2011), no. 9, 2568-2597.

[9] - Torus action on $S^{n}$ and sign changing solutions for conformally invariant equations, Ann. Sc. Norm. Super. Pisa Cl. Sci. (5). To appear.

[10] W. Y. Ding, On a conformally invariant elliptic equation on $\mathbb{R}^{n}$, Comm. Math. Phys. 107 (1986), no. 2, 331-335.

[11] O. Druet, From one bubble to several bubbles: the low-dimensional case, J. Differential Geom. 63 (2003), no. 3, 399-473.

[12] Compactness for Yamabe metrics in low dimensions, Int. Math. Res. Not. 23 (2004), $1143-1191$.

[13] O. Druet and E. Hebey, Stability for strongly coupled critical elliptic systems in a fully inhomogeneous medium, Anal. PDE 2 (2009), no. 3, 305-359.

[14] O. Druet, E. Hebey, and F. Robert, Blow-up theory for elliptic PDEs in Riemannian geometry, Mathematical Notes, vol. 45, Princeton University Press, Princeton, NJ, 2004.

[15] O. Druet, E. Hebey, and J. Vétois, Bounded stability for strongly coupled critical elliptic systems below the geometric threshold of the conformal Laplacian, J. Funct. Anal. 258 (2010), no. 3, 999-1059.

[16] P. Esposito, A. Pistoia, and J. Vétois, The effect of linear perturbations on the Yamabe problem. Preprint.

[17] Y. Guo, B. Li, and J.-C. Wei, Large energy entire solutions for the Yamabe-type problem of polyharmonic operator, J. Differential Equations. To appear.

[18] E. Hebey and J.-C. Wei, Resonant states for the static Klein-Gordon-Maxwell-Proca system, Math. Res. Lett. to appear.

[19] M. A. Khuri, F. C. Marques, and R. M. Schoen, A compactness theorem for the Yamabe problem, J. Differential Geom. 81 (2009), no. 1, 143-196. 
[20] J.L. Kazdan and F.W. Warner, Scalar curvature and conformal deformation of Riemannian structure, J. Differential Geometry 10 (1975), 113-134.

[21] J.M. Lee and T.H. Parker, The Yamabe problem, Bull. Amer. Math. Soc. (N.S.) 17 (1987), no. 1, 37-91.

[22] Y. Y. Li and L. Zhang, A Harnack type inequality for the Yamabe equation in low dimensions, Calc. Var. Partial Differential Equations 20 (2004), no. 2, 133-151.

[23] _ Compactness of solutions to the Yamabe problem. II, Calc. Var. Partial Differential Equations 24 (2005), no. 2, 185-237.

[24] Y.Y. Li and L. Zhang, Compactness of solutions to the Yamabe problem. III, J. Funct. Anal. 245 (2007), no. 2, 438-474.

[25] Y. Y. Li and M. J. Zhu, Yamabe type equations on three-dimensional Riemannian manifolds, Commun. Contemp. Math. 1 (1999), no. 1, 1-50.

[26] F. C. Marques, A priori estimates for the Yamabe problem in the non-locally conformally flat case, J. Differential Geom. 71 (2005), no. 2, 315-346.

[27] A. M. Micheletti, A. Pistoia, and J. Vétois, Blow-up solutions for asymptotically critical elliptic equations, Indiana Univ. Math. J. 58 (2009), no. 4, 1719-1746.

[28] M. Obata, The conjectures on conformal transformations of Riemannian manifolds, J. Differential Geometry 6 (1971/72), 247-258.

[29] A. Pistoia and J. Vétois, Sign-changing bubble towers for asymptotically critical elliptic equations on Riemannian manifolds (2012). Preprint.

[30] O. Rey, The role of the Green's function in a nonlinear elliptic equation involving the critical Sobolev exponent, J. Funct. Anal. 89 (1990), no. 1, 1-52.

[31] E. Rodemich, The Sobolev inequalities with best possible constants, Analysis Seminar at California Institute of Technology (1966).

[32] R. M. Schoen, Variational theory for the total scalar curvature functional for Riemannian metrics and related topics, Topics in calculus of variations (Montecatini Terme, 1987), Lecture Notes in Math., vol. 1365, Springer, Berlin, 1989, pp. 120-154.

[33] _ On the number of constant scalar curvature metrics in a conformal class, Differential geometry, Pitman Monogr. Surveys Pure Appl. Math., vol. 52, Longman Sci. Tech., Harlow, 1991, pp. 311-320.

[34] M. Struwe, A global compactness result for elliptic boundary value problems involving limiting nonlinearities, Math. Z. 187 (1984), no. 4, 511-517.

[35] G. Talenti, Best constant in Sobolev inequality, Ann. Mat. Pura Appl. (4) 110 (1976), 353372.

[36] J. Vétois, Multiple solutions for nonlinear elliptic equations on compact Riemannian manifolds, Internat. J. Math. 18 (2007), no. 9, 1071-1111.

[37] J.-C. Wei and S. Yan, On a stronger Lazer-McKenna conjecture for Ambrosetti-Prodi type problems, Ann. Sc. Norm. Super. Pisa Cl. Sci. (5) 9 (2010), no. 2, 423-457.

Frédéric Robert, Institut Élie Cartan, Université de Lorraine, BP 239, F-54506 VANDCEUVRE-LÈS-NANCY, France

E-mail address: frederic.robert@univ-lorraine.fr

Jérôme Vétois, Université de Nice Sophia Antipolis, laboratoire J.-A. Dieudonné, CNRS UMR 6621, Parc Valrose, F-06108 Nice Cedex 2, France

E-mail address: vetois@unice.fr 